Review Article

\title{
Confocal Laser Endomicroscopy in Gastrointestinal and Pancreatobiliary Diseases: A Systematic Review and Meta-Analysis
}

\author{
Alessandro Fugazza, ${ }^{1}$ Federica Gaiani, ${ }^{1}$ Maria Clotilde Carra, ${ }^{2}$ \\ Francesco Brunetti, ${ }^{3}$ Michaël Lévy, ${ }^{4}$ Iradj Sobhani, ${ }^{4,5}$ Daniel Azoulay, ${ }^{3}$ \\ Fausto Catena, ${ }^{6}$ Gian Luigi de'Angelis, ${ }^{1}$ and Nicola de'Angelis ${ }^{3,5,7}$ \\ ${ }^{1}$ Unit of Gastroenterology and Digestive Endoscopy, University of Parma, 43100 Parma, Italy \\ ${ }^{2}$ University Paris VII-Denis Diderot, 75006 Paris, France \\ ${ }^{3}$ Unit of Digestive, Hepato-Pancreato-Biliary Surgery and Liver Transplantation, Henri Mondor Hospital, AP-HP, 94010 Créteil, France \\ ${ }^{4}$ Department of Gastroenterology and Digestive Endoscopy, Henri Mondor Hospital, AP-HP, 94010 Créteil, France \\ ${ }^{5}$ Cancer Research Lab. EC2M3, Université Paris-Est, Val de Marne UPEC, 94010 Créteil, France \\ ${ }^{6}$ Emergency Surgery Department, University of Parma, 43100 Parma, Italy \\ ${ }^{7}$ Department of Advanced Biomedical Sciences, University Federico II of Naples, 80138 Naples, Italy
}

Correspondence should be addressed to Alessandro Fugazza; alessandro.fugazza@studenti.unipr.it

Received 6 September 2015; Accepted 31 December 2015

Academic Editor: Gerald J. Wyckoff

Copyright (C) 2016 Alessandro Fugazza et al. This is an open access article distributed under the Creative Commons Attribution License, which permits unrestricted use, distribution, and reproduction in any medium, provided the original work is properly cited.

Confocal laser endomicroscopy (CLE) is an endoscopic-assisted technique developed to obtain histopathological diagnoses of gastrointestinal and pancreatobiliary diseases in real time. The objective of this systematic review is to analyze the current literature on CLE and to evaluate the applicability and diagnostic yield of CLE in patients with gastrointestinal and pancreatobiliary diseases. A literature search was performed on MEDLINE, EMBASE, Scopus, and Cochrane Oral Health Group Specialized Register, using pertinent keywords without time limitations. Both prospective and retrospective clinical studies that evaluated the sensitivity, specificity, or accuracy of CLE were eligible for inclusion. Of 662 articles identified, 102 studies were included in the systematic review. The studies were conducted between 2004 and 2015 in 16 different countries. CLE demonstrated high sensitivity and specificity in the detection of dysplasia in Barrett's esophagus, gastric neoplasms and polyps, colorectal cancers in inflammatory bowel disease, malignant pancreatobiliary strictures, and pancreatic cysts. Although CLE has several promising applications, its use has been limited by its low availability, high cost, and need of specific operator training. Further clinical trials with a particular focus on cost-effectiveness and medicoeconomic analyses, as well as standardized institutional training, are advocated to implement CLE in routine clinical practice.

\section{Introduction}

Confocal laser endomicroscopy (CLE) is an endoscopic modality that was developed to obtain very high magnification of the mucosal layer of the gastrointestinal (GI) tract [1], and it has the potential to enable histological diagnosis in real time [2]. CLE is based on tissue illumination using a lowpower laser and the subsequent detection of fluorescent light that is reflected back from the tissue through a pinhole [3]. The term "confocal" refers to the alignment of both illumination and collection systems in the same focal plane $[4,5]$. In brief, the laser light is focused at a selected depth in the tissue of interest and reflected light is then refocused onto the detection system by the same lens. Only the returning light that is refocused through the pinhole is detected. Any light that is reflected or scattered at other geometric angles 
from the illuminated object or any light that is refocused out of plane with the pinhole is excluded from detection. This dramatically increases the spatial resolution of CLE and enables cellular imaging and evaluation of tissue architecture at the focal plane to be performed during endoscopy [1].

To obtain confocal images, exogenous fluorescence agents can be administered either topically or systemically [6]. The most common topical contrast agents that are applied by a spraying catheter are acriflavine and cresyl violet [7], whereas the most widely used systemically administered fluorescent agent is intravenous fluorescein sodium. Fluoresceins are nontoxic, and their administration has been associated with only rare adverse events, including transient hypotension without shock $(0.5 \%)$, nausea $(0.39 \%)$, injection site erythema $(0.35 \%)$, self-limited diffuse rash $(0.04 \%)$, and mild epigastric pain $(0.09 \%)$ [8].

There are two types of CLE: endoscope-based (eCLE) and probe-based (pCLE) endomicroscopy. To perform eCLE, a dedicated endoscope with a miniaturized confocal scanner integrated into the distal tip is employed [9]. This system was developed by Pentax (Tokyo, Japan) and enables simultaneous endoscopic imaging to be performed. Additionally, it frees the endoscopic working channel, and it can be used for targeted biopsies or combined enhancement techniques $[9,10]$. Images can be collected by sectioning down through the mucosa in $7 \mu \mathrm{m}$ increments to a depth of $250 \mu \mathrm{m}$. Image stabilization is necessary to obtain good quality images [11]. The second system is pCLE (Cellvizio, Mauna Kea Technologies, Paris, France). In this system, confocal miniprobes can be passed down the accessory channel of any standard endoscope [12], providing rapid image capture and "stitching" of adjacent images to create a "mosaic image" in real time [11]. The advantages of pCLE are its versatility and the possibility of combining it with other imaging modalities such as virtual chromoendoscopy or magnification [9]. Image stabilization can be achieved by using a plastic cap on the endoscope tip. There are several types of probes that are characterized by different imaging depths, fields of view, and lateral resolutions (Supplementary Table S1 in Supplementary Material available online at http://dx.doi.org/10.1155/2016/4638683). More recently, a novel needle-based CLE ( $\mathrm{nCLE}$ ) miniprobe (AQFlex 19; Mauna Kea) has been developed which can pass through a $19 \mathrm{G}$ needle to perform endoscopic ultrasoundguided fine-needle aspiration (EUS-FNA) on solid organs and lymph nodes [13-16]. The probes can be reused after disinfection for as many as 10 to 20 examinations [1].

CLE can be applied to examine luminal structures, such as esophagus, stomach, and colon, and ductal structures, such as bile and pancreatic ducts. Ultimately, CLE can be used to optimize endoscopic diagnoses, thereby reducing unnecessary resections, avoiding repeated biopsies and unnecessary follow-up, and indirectly decreasing the risks and costs that are associated with repeated indiscriminative endoscopic exams. However, the interpretation of CLE images is still challenging. A standard classification system for distinguishing between normal and pathological GI conditions has only been developed for p-CLE devices; it was termed the Miami Classification, and it was based on a consensus that was reached among $\mathrm{p}$-CLE users during a meeting that was held in Miami in 2009 [12]. Conversely, international recommendations and guidelines for other CLE systems have not yet been assessed.

The objective of the present systematic review is to summarize and analyze the current literature evaluating the sensitivity and specificity of this novel imaging modality (i.e., CLE) in detecting mucosal abnormalities. In assessing the available literature, we aimed to highlight the utility of CLE in the diagnosis of gastrointestinal and pancreatobiliary diseases, particularly in the screening or surveillance of dysplastic and neoplastic lesions, and to consider its potential future impact on daily clinical practice.

\section{Methods}

The methodological approach included the definition of search strategies, the development of selection criteria, an assessment of study quality, and the abstraction of relevant data. The PRISMA statements checklist for systematic review reporting was followed.

2.1. Study Inclusion Criteria. The study selection criteria were defined prior to initiating data collection to enable the proper identification of eligible studies for inclusion in the analysis.

The search was restricted to studies that were performed in humans and that were published in English. Prospective and retrospective clinical studies were both eligible for inclusion, and there were no limits based on trial duration. Review articles, case reports, commentaries, editorials, letters, and conference abstracts were not considered. Likewise, ex vivo studies were excluded. Endoscopic applications of CLE were only considered if they were being used to evaluate the following types of diseases/lesions: Barrett's esophagus; squamous cell carcinoma; esophagitis; gastroesophageal reflux disease; gastric polyps; gastric atrophy and reactive metaplasia, dysplasia, and neoplasia; Helicobacter pylori-related gastritis; inflammatory bowel disease (IBD); celiac disease; colonic neoplasm; colonic polyps; biliary duct disease; and benign or malignant pancreatic diseases. To be eligible for inclusion, a study must have included at least one of the following outcomes: sensitivity, specificity, positive predictive value (PPV), negative predictive value (NPV), accuracy, description of CLE indications, or mucosal features found using CLE.

2.2. Literature Search Strategy. A literature search was performed using the following online databases: MEDLINE (through PubMed), EMBASE, Scopus, and Cochrane Oral Health Group Specialized Register. A grey literature search was also performed by using the OpenGrey database. To increase the probability of identifying all relevant articles, a specific research equation was formulated for each database, using the following key words and/or MeSH terms: confocal laser endomicroscopy, CLE, endomicroscopy, gastrointestinal, esophagus, esophageal, bile duct, biliary, gastric, colon, colic, pancreatic, and pancreas. Additionally, reference lists from eligible studies and relevant review articles (not included in the systematic review) were cross-checked to identify additional studies. 
2.3. Study Selection and Quality Assessment. The titles and abstracts of the retrieved studies were independently and blindly screened for relevance by two reviewers (Alessandro Fugazza and Federica Gaiani). A full article was retrieved when the information in the title and/or abstract appeared to meet the objective of this review. To enhance sensitivity, records were removed only when both reviewers excluded the record at the title screening level. All disagreements were resolved through discussions with a third and fourth reviewer (Nicola de'Angelis, Michaël Lévy). Subsequently, two reviewers (Alessandro Fugazza, Federica Gaiani) independently performed a full-text analysis and quality assessment of the selected articles. The Cochrane criteria, which are described in the Cochrane Handbook for Systematic Reviews of Interventions [17], were applied for randomized clinical trials, and the Newcastle-Ottawa Scale (NOS) [18] was used for nonrandomized studies.

2.4. Data Extraction and Analysis. The data that were extracted from the studies for inclusion in the systematic review were processed for qualitative and possibly quantitative analyses. The data were collected and summarized based on whether CLE was applied for gastrointestinal or pancreatobiliary diseases. Data that would enable us to perform "per patient," "per biopsy," and "per lesion" analyses were separately extracted whenever available. A "per patient" analysis was performed by comparing each patient's final CLE outcome against their histopathological diagnosis; similarly, "per biopsy" and "per lesion" analyses were conducted by comparing images produced by CLE with the results of histologic biopsies. All data extraction was performed by one author (Alessandro Fugazza) and was verified by a second author (Federica Gaiani), and a 100\% rate of final agreement was maintained.

To perform quantitative analysis, data on sensitivity, specificity, PPV, NPV, and main findings were extracted from the eligible studies. If necessary and possible, outcome variables were calculated by the authors based on the data that were available in individually selected studies. Only on-site (i.e., real-time) data of CLE imaging analysis were considered. Meta-analyses were performed using MetaDisc (version 5.2; Clinical Biostatistics Unit, Hospital Ramon y Cajal, Madrid, Spain) [19]. Heterogeneity was assessed using $I^{2}$ statistics.

\section{Results}

All database searches were performed without time limit until January 2015. Overall, the combined search identified 662 articles (after removing duplicates); of these, 348 were rejected based upon title and abstract evaluation. Out of the remaining articles, which underwent full-text evaluations, 212 were excluded because they either were not pertinent to the review questions, had nonrelevant study designs, or had language limitations. A final total of 102 articles were considered to be eligible for the systematic review and were evaluated by both qualitative and quantitative analyses. A flowchart of the study's identification and inclusion/exclusion processes is shown in Figure 1.
3.1. Study Characteristics. The studies that were included were published between 2004 and 2015. They included 8 RCTs, 85 prospective studies, and 9 retrospective studies. Twenty-eight (27.4\%) studies were multicentric, with a maximum of 8 centers included. The studies were conducted in 16 different countries. The included single-center studies were conducted in Asia $(n=33)$, Europe $(n=31)$, USA $(n=7)$, and Oceania $(n=3)$; the multicentric studies were conducted in Europe + USA $(n=15)$, Europe + Asia $(n=2)$, Europe $(n=5)$, and the USA $(n=6)$.

Overall, the 102 included studies enrolled a total of 6943 patients; the number of patients per study ranged from 4 to 1572 , with a median sample of 67.4 patients. The median patient age was 56.9 years (range: 0.7 to 90 years). All of the patients that underwent CLE were administered intravenous fluorescein prior to the procedure.

The outcomes produced by CLE technology are hereafter classified by organ of application, including esophagus, stomach, pancreas, biliary tree, and colon.

3.2. Esophagus. The most important application of CLE in the esophagus is the surveillance and evaluation of suspicious lesions in patients presenting with Barrett's esophagus (BE) [20-31]. With regard to the detection of premalignant and malignant transformations of metaplasia in BE patients, a "per biopsy" meta-analysis of 7 studies [20, 21, 23-26, 30] resulted in a pooled sensitivity of $58 \%\left(\mathrm{CI}_{95 \%}\right.$ : $52 \%-63 \%$; $\left.I^{2}: 95.2 \%\right)$, a pooled specificity of $90 \%\left(\mathrm{CI}_{95 \%}: 89 \%-91 \%\right.$; $\left.I^{2}: 96.9 \%\right)$, a pooled positive likelihood ratio (LR) of 11.57 $\left(\mathrm{CI}_{95 \%}: 5.38-24.89 ; I^{2}: 93.7 \%\right)$, and a pooled negative LR of $0.23\left(\mathrm{CI}_{95 \%}: 0.08-0.64 ; I^{2}: 98 \%\right)$. The area under the curve was 0.9758. A "per patient" meta-analysis based on 4 studies [21, $24,30,31]$ yielded a pooled sensitivity of $79 \%\left(\mathrm{CI}_{95 \%}: 65 \%-\right.$ $\left.90 \% ; I^{2}: 58.5 \%\right)$, a pooled specificity of $90 \%\left(\mathrm{CI}_{95 \%}: 85 \%-94 \%\right.$; $\left.I^{2}: 82.9 \%\right)$, a pooled positive LR of $8.04\left(\mathrm{CI}_{95 \%}: 2.28-28.3 ; I^{2}\right.$ : $83.5 \%)$, and a pooled negative LR of $0.24\left(\mathrm{CI}_{95 \%}: 0.08-0.69\right.$; $I^{2}: 55.4 \%$ ). The area under the curve was 0.926 (Figure 2 and Table 1). Only anecdotal reports have described using CLE to detect the features of squamous cell carcinoma [32-34], reflux esophagitis [35], and nonerosive reflux disease (NERD) [36]. These latter studies were not included in the quantitative analysis (Table 1).

3.3. Stomach and Duodenum. The use of CLE in patients presenting with gastric disease has raised great interest, particularly in Asian countries, where these pathologies are highly prevalent. The primary applications of CLE with respect to the stomach and duodenum have included the detection of polyps and neoplastic lesions and the study of gastritis and metaplastic lesions [37, 41, 43, 44, 46, 47, 49, 51, 52, 54, 56]; the application of CLE has also shown utility in patients with celiac disease [60-62] and Helicobacter pylori-related gastritis [58] (Table 2). The performance of this innovative technique has been evaluated both alone and in comparison or in addition to other methods, such as endoscopic ultrasound (EUS) and narrow band imaging (NBI); however, the majority of these studies had descriptive aims and focused on the utility of employing CLE in targeting biopsies [40,48]. With respect 


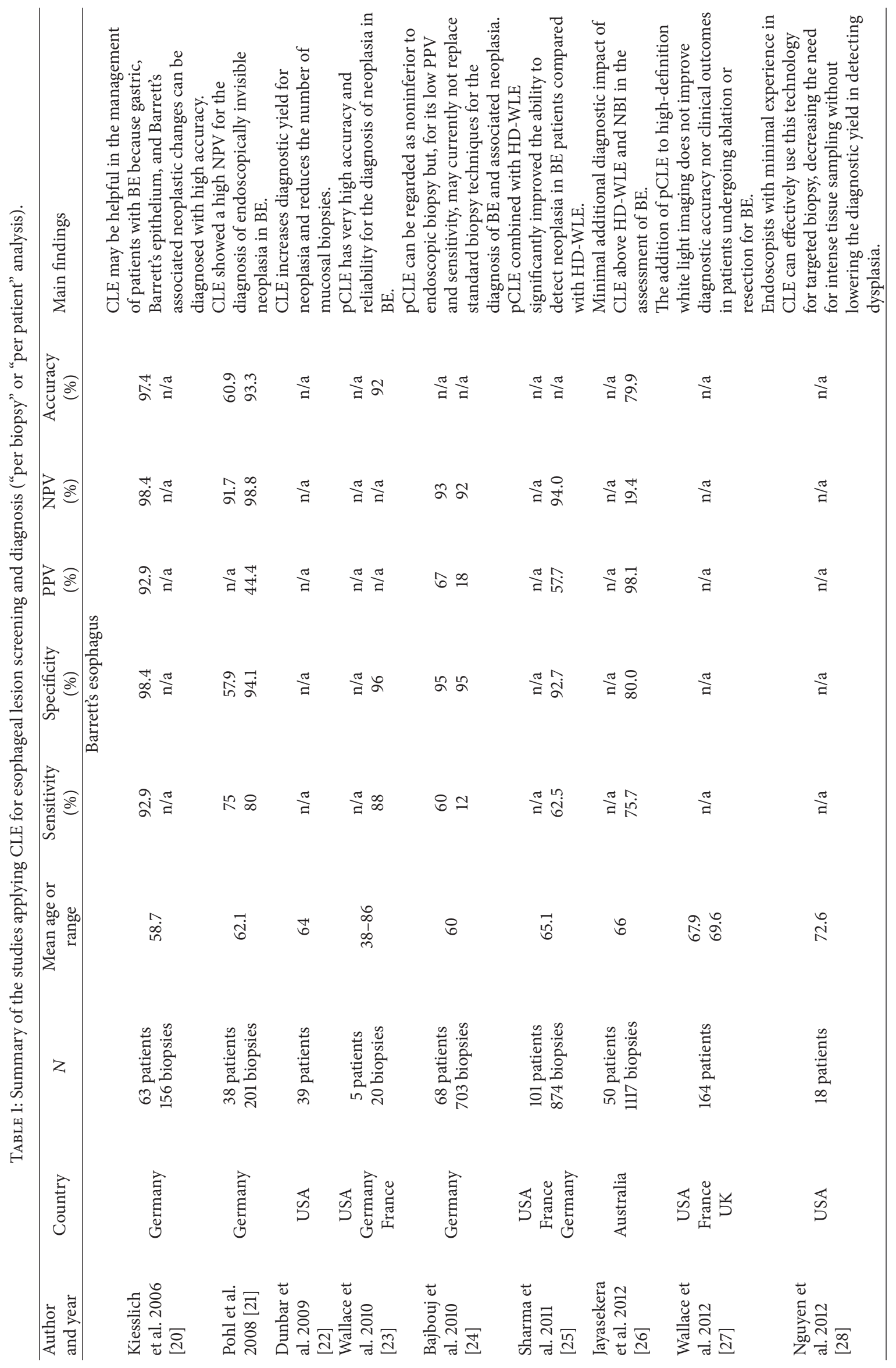




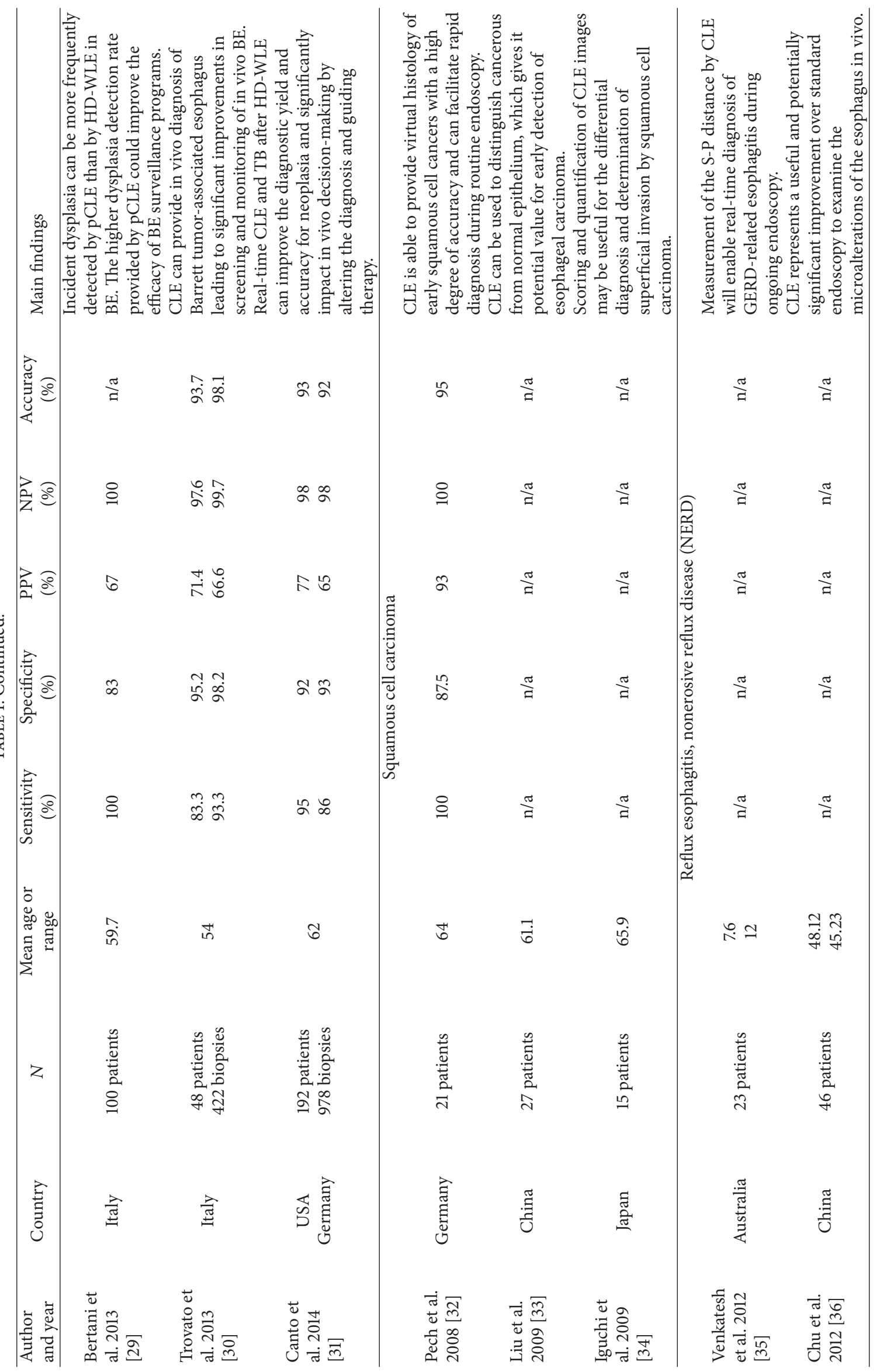




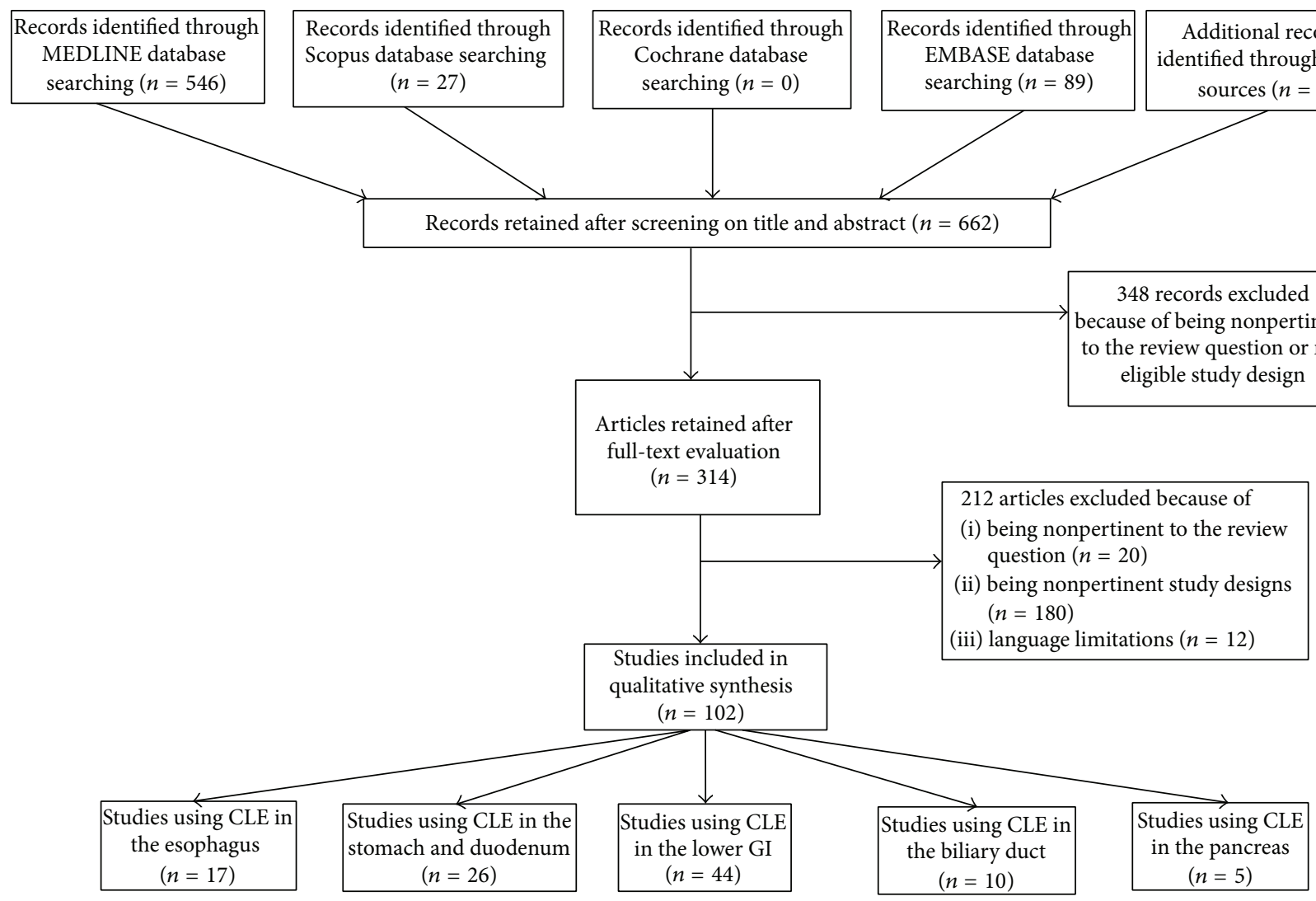

FIGURE 1: Flow chart of the electronic literature search strategy using Medline, Scopus, Embase, and other sources.

to the detection and diagnosis of polyps and neoplastic lesions, a "per patient" meta-analysis was only possible for 3 of the included studies $[43,45,46]$, and it yielded a pooled sensitivity of $85 \%\left(\mathrm{CI}_{95 \%}: 78 \%-91 \% ; I^{2}: 52.3 \%\right)$, a pooled specificity of $99 \%\left(\mathrm{CI}_{95 \%}: 98 \%-99 \% ; I^{2}: 92.9 \%\right)$, a pooled positive LR of $16.49\left(\mathrm{CI}_{95 \%}: 1.48-183.19 ; I^{2}: 96 \%\right)$, and a pooled negative LR of $0.16\left(\mathrm{CI}_{95 \%}: 0.08-0.35 ; I^{2}: 57.4 \%\right)$ (Figure 3 ). The area under the curve was 0.929 . The estimated diagnostic accuracy of CLE ranged from $85 \%$ to $98.8 \%$. With respect to the study of gastritis and gastric metaplasia, 6 studies $[50,51,53-56]$ were included in the "per biopsy" metaanalysis, which resulted in a pooled sensitivity of $94 \%\left(\mathrm{CI}_{95 \%}\right.$ : $\left.92 \%-96 \% ; I^{2}: 54.8 \%\right)$, a pooled specificity of $95 \%\left(\mathrm{CI}_{95 \%}\right.$ : $\left.92 \%-97 \% ; I^{2}: 55.6 \%\right)$, a positive LR of $17.66\left(\mathrm{CI}_{95 \%}: 9.04-\right.$ $\left.34.51 ; I^{2}: 63.8 \%\right)$, and a negative LR of $0.07\left(\mathrm{CI}_{95 \%}: 0.04-0.12\right.$; $\left.I^{2}: 47.4 \%\right)$. The area under the curve was 0.9832 (Figure 4).

A meta-analysis of two studies regarding Helicobacter Pylori-related gastritis $[57,58]$ yielded a pooled sensitivity of $86 \%\left(\mathrm{CI}_{95 \%}: 76 \%-93 \% ; I^{2}: 0 \%\right)$, a pooled specificity of $93 \%$ $\left(\mathrm{CI}_{95 \%}: 87 \%-97 \% ; I^{2}: 2.6 \%\right)$, a positive $\mathrm{LR}$ of $11.28\left(\mathrm{CI}_{95 \%}: 5.4-\right.$ $\left.23.57 ; I^{2}: 15.5 \%\right)$, and a negative $\mathrm{LR}$ of $0.16\left(\mathrm{CI}_{95 \%}: 0.09-0.27\right.$; $I^{2}: 0 \%$ ) (Figure S1).

The use of CLE in assessing celiac disease, with respect to intraepithelial lymphocytes and villous atrophy, was proven to have high sensitivity and specificity. A meta-analysis performed on 3 studies [60-62] produced a pooled sensitivity of $84 \%\left(\mathrm{CI}_{95 \%}: 72 \%-92 \% ; I^{2}: 71.3 \%\right)$, a pooled specificity of $94 \%\left(\mathrm{CI}_{95 \%}: 85 \%-99 \% ; I^{2}: 66.4 \%\right)$, a positive LR of $9.9\left(\mathrm{CI}_{95 \%}\right.$ : $\left.2.12-46.35 ; I^{2}: 53.9 \%\right)$, and a negative LR of $0.15\left(\mathrm{CI}_{95 \%}: 0.04-\right.$ $\left.0.52 ; I^{2}: 45.2 \%\right)$. The area under the curve was 0.9691 (Figure S2).

3.4. Colon. There is a wide range of applications for the use of CLE in patients with colonic diseases. These include the description of elementary and pathognomonic lesions in IBD [63-75], the detection of dysplasia/neoplasia in healed mucosa in IBD patients [76-81], and the microscopic description of colorectal polypoid lesions and neoplasms [82-102]. Although they have been less extensively studied, additional applications of CLE include obtaining microscopic descriptions of mucosa in patients with irritable bowel syndrome (IBS) $[105,106]$, infectious colitis (i.e., clostridium difficile infection) [104], and colitis associated with Graft versus Host Disease (GVHD) [103] (Table 3).

A meta-analysis of 4 studies [77, 79-81] that investigated dysplasia and neoplasia in IBD patients produced a pooled "per lesion" sensitivity of $80 \%\left(\mathrm{CI}_{95 \%}: 61 \%-92 \% ; I^{2}: 84.5 \%\right)$, a pooled specificity of $93 \%\left(\mathrm{CI}_{95 \%}: 89 \%-96 \% ; I^{2}: 86.3 \%\right)$, a pooled positive LR of $8.76\left(\mathrm{CI}_{95 \%}: 1.78-44.23 ; I^{2}: 71.7 \%\right)$, and a pooled negative $\mathrm{LR}$ of $0.25\left(\mathrm{CI}_{95 \%}: 0.01-7.44 ; I^{2}: 96.2 \%\right)$. The area under the curve was 0.9630 (Figure 5). A meta-analysis of 7 studies that investigated colorectal neoplasms and polyps 


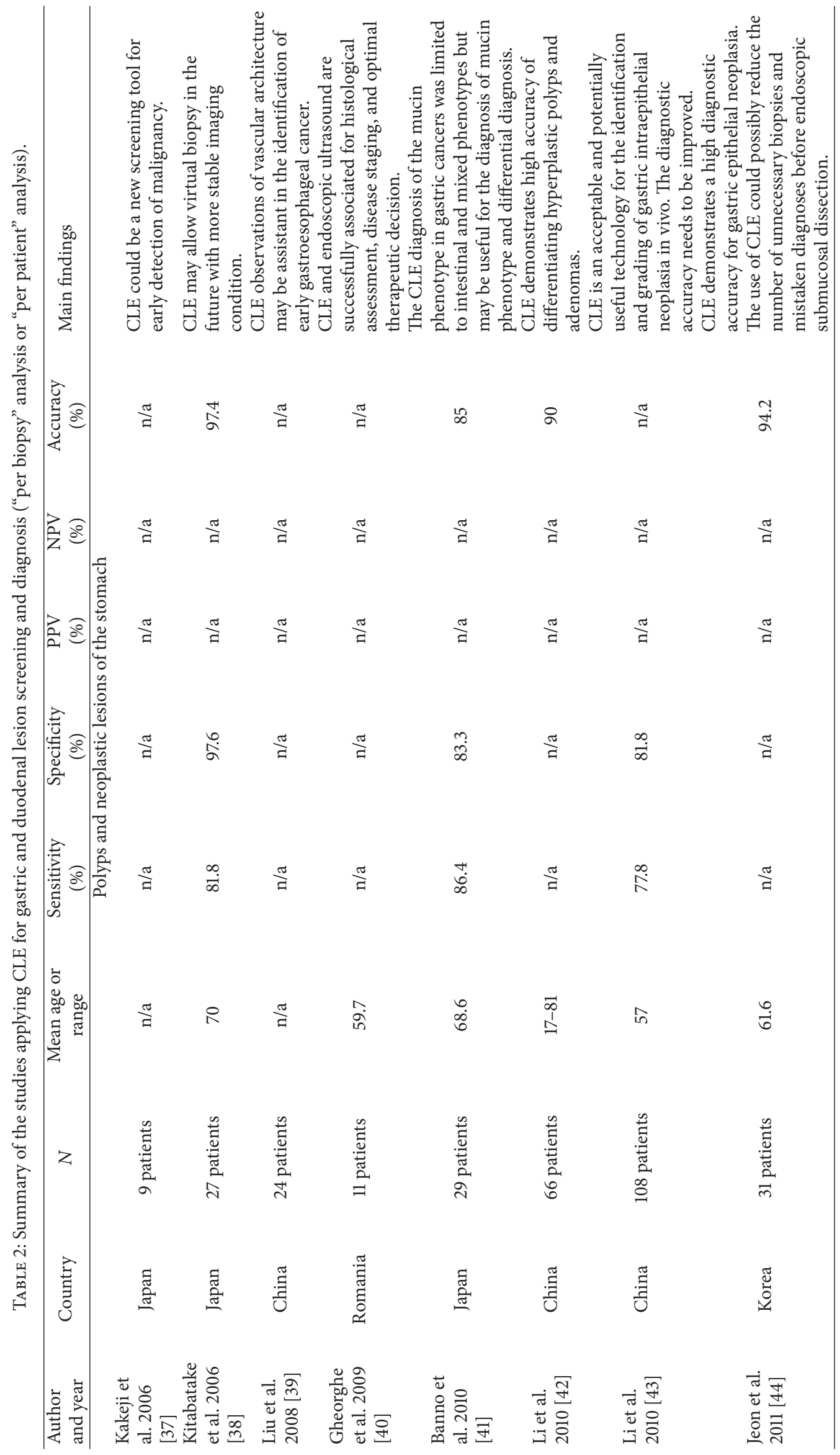




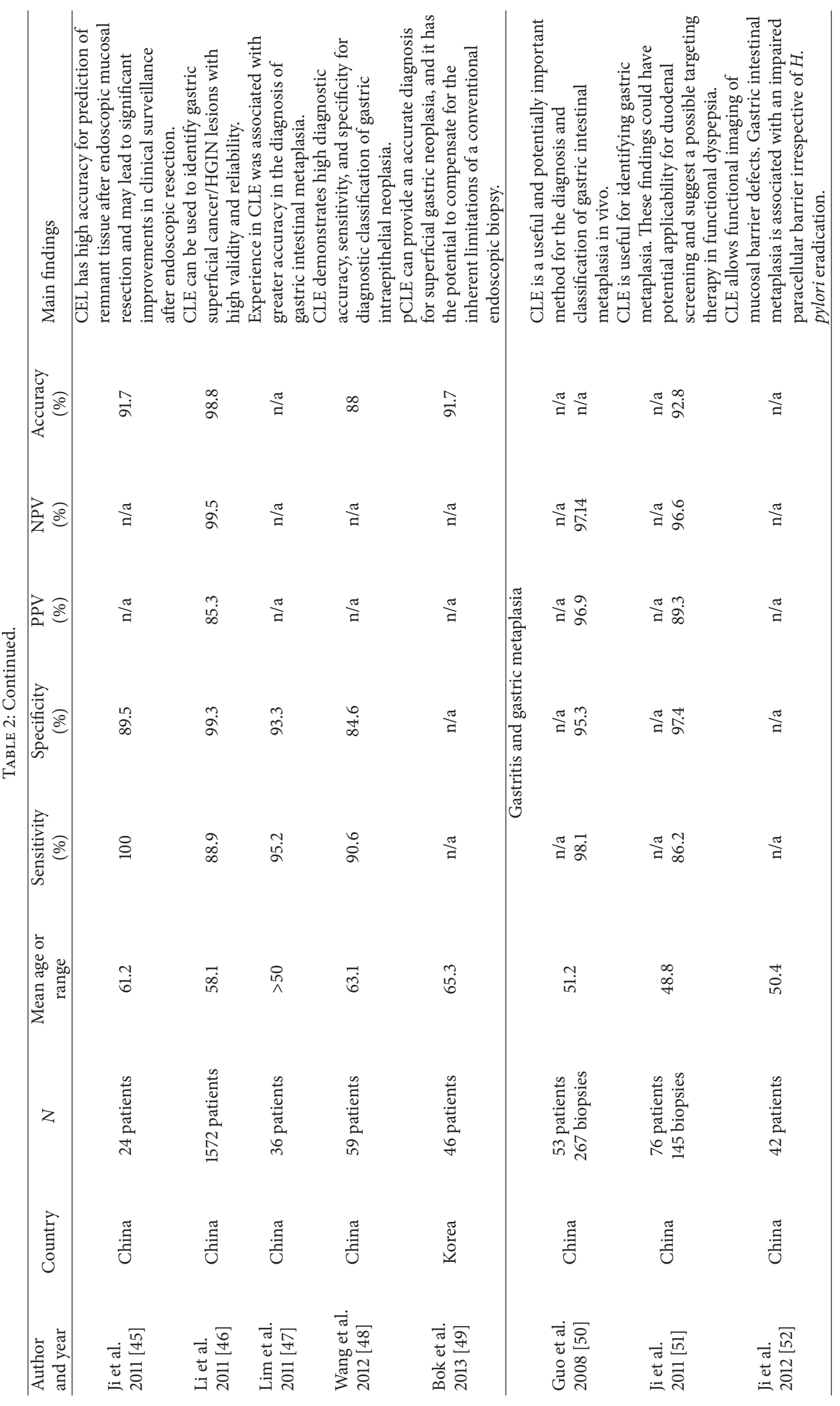




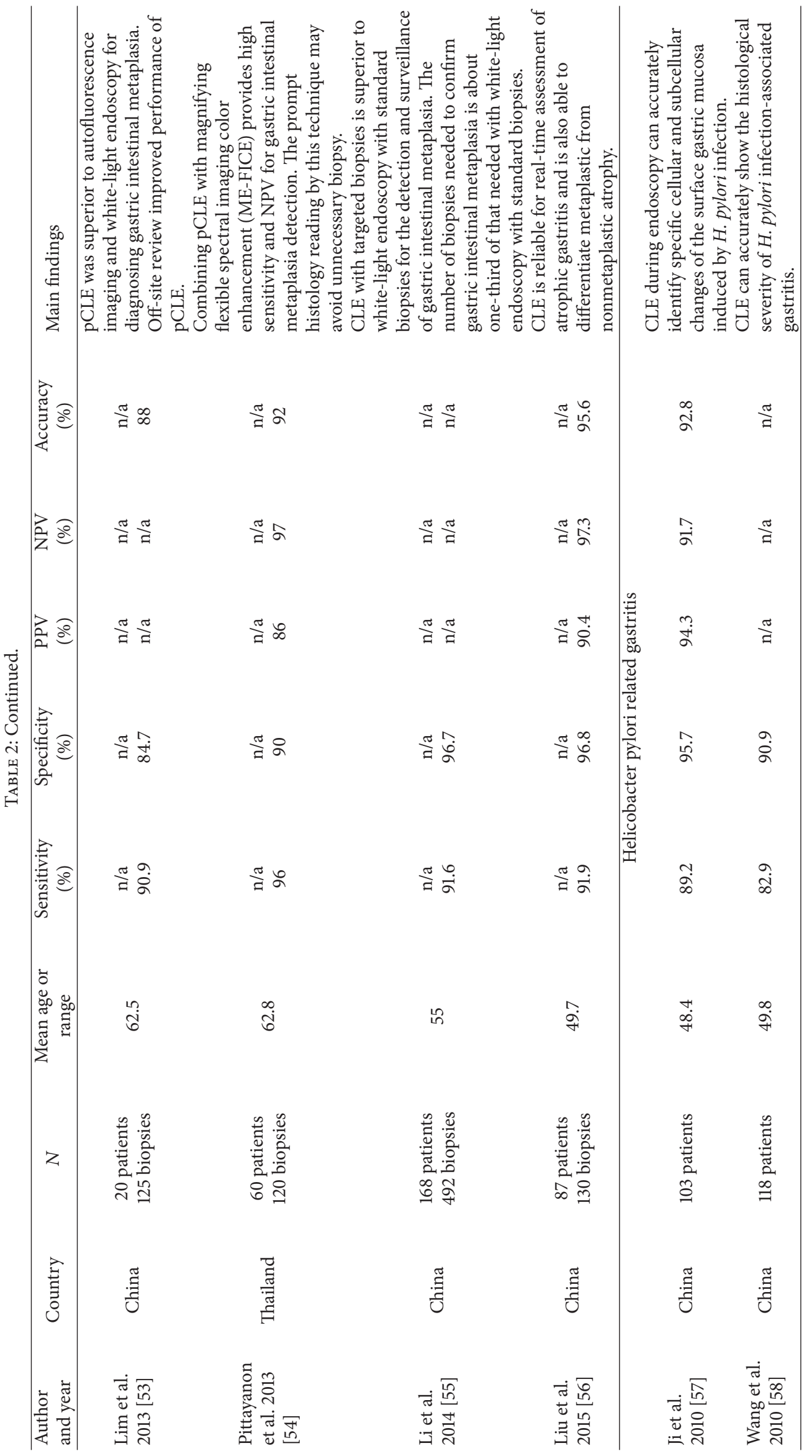




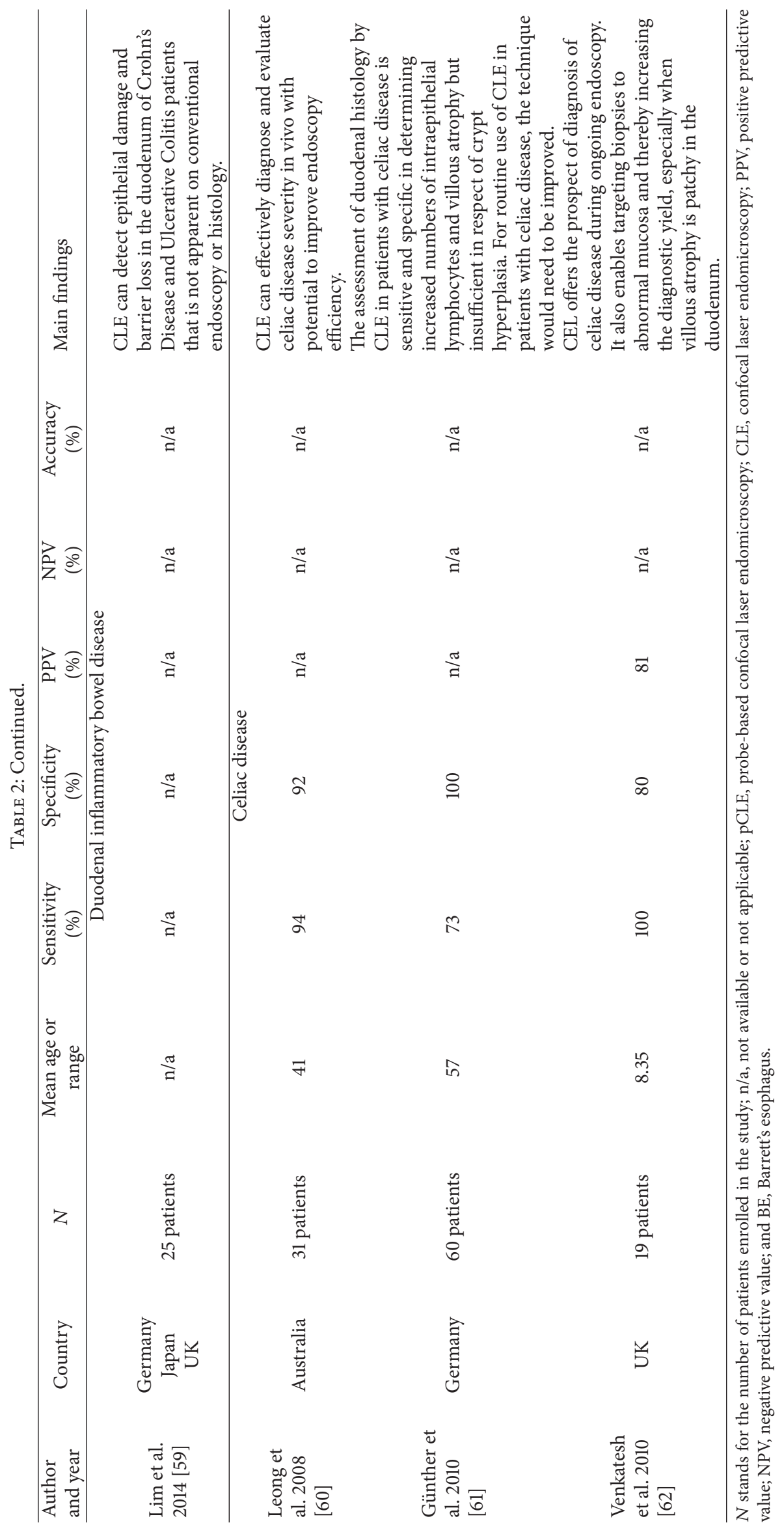




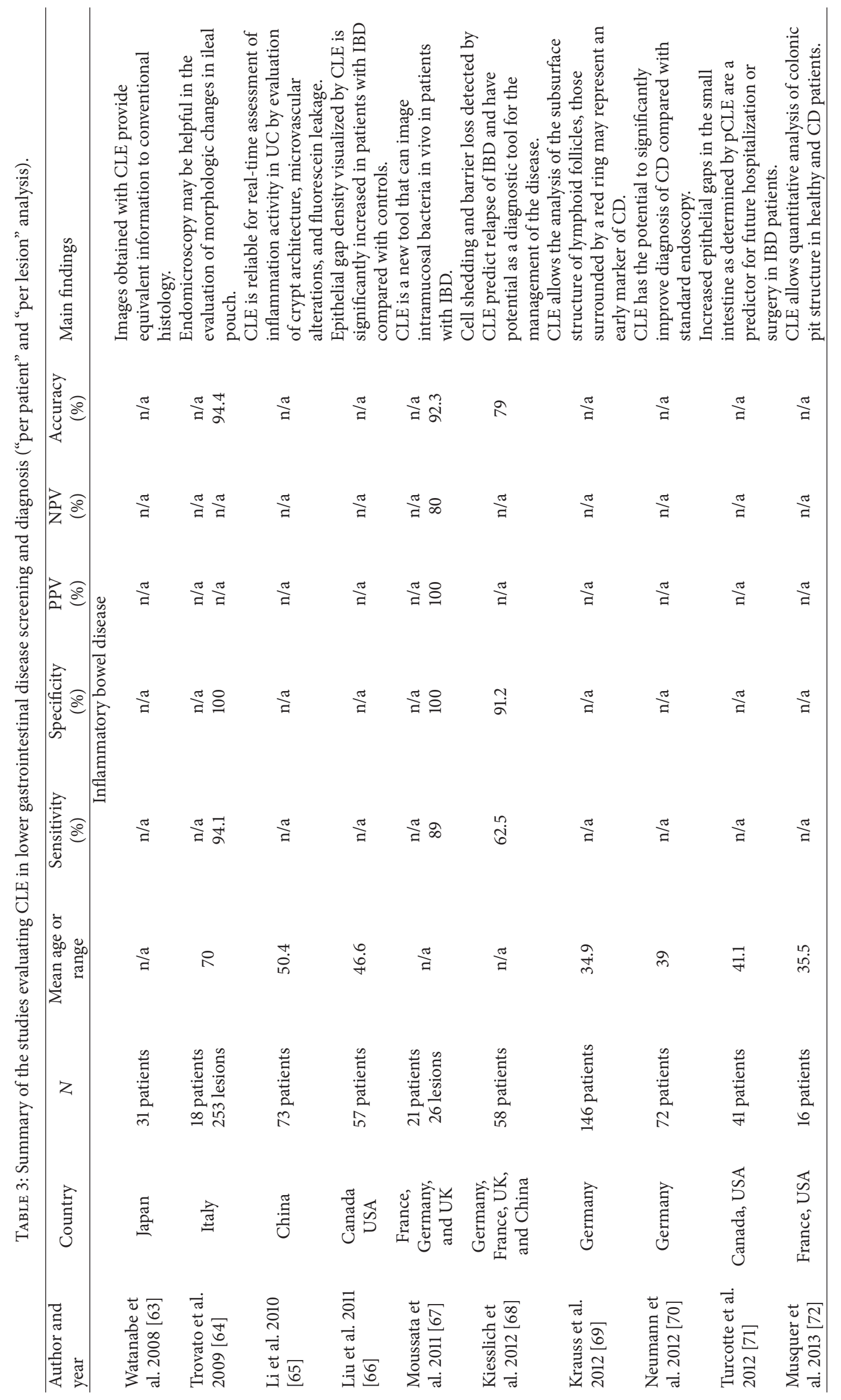




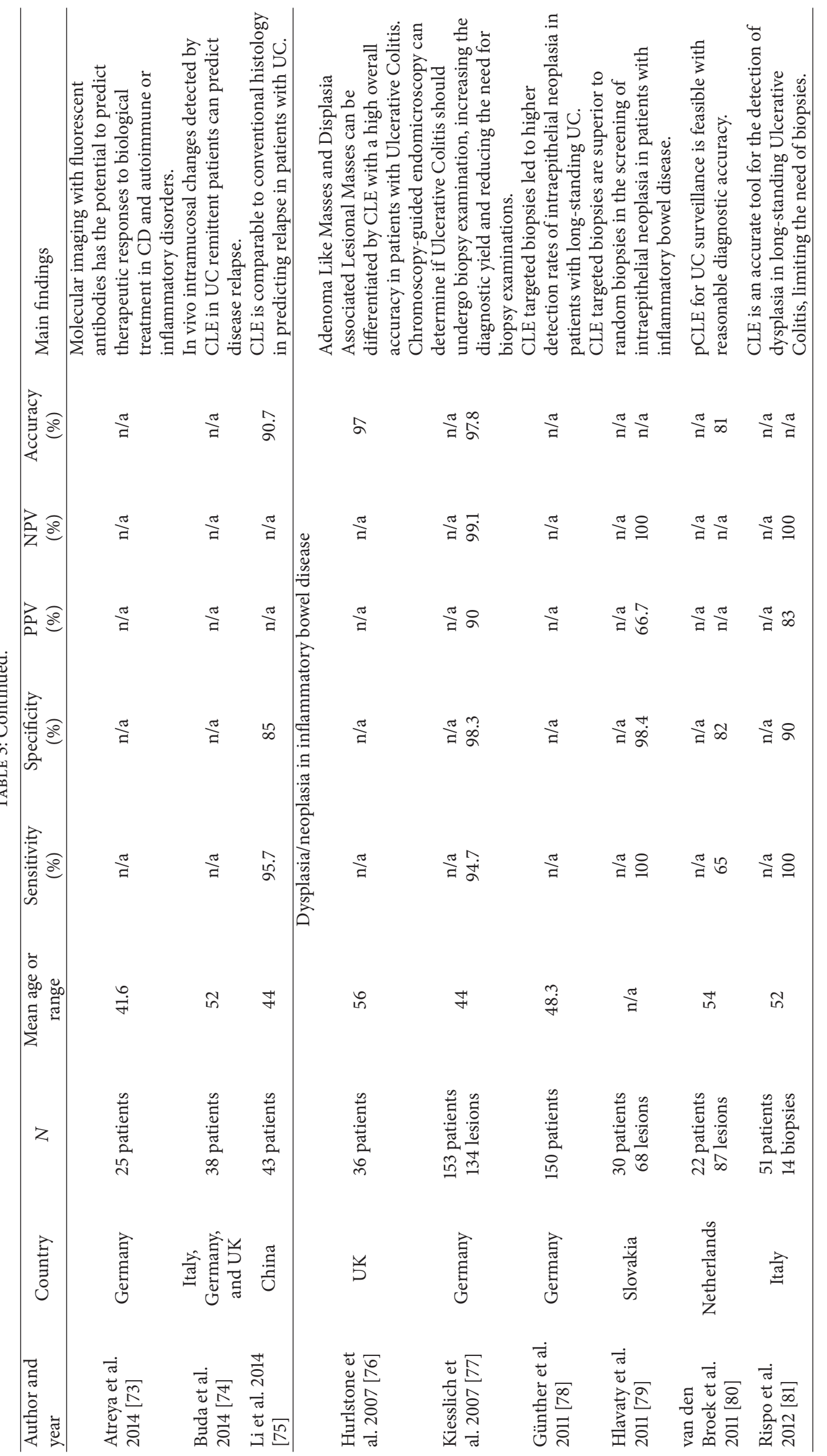




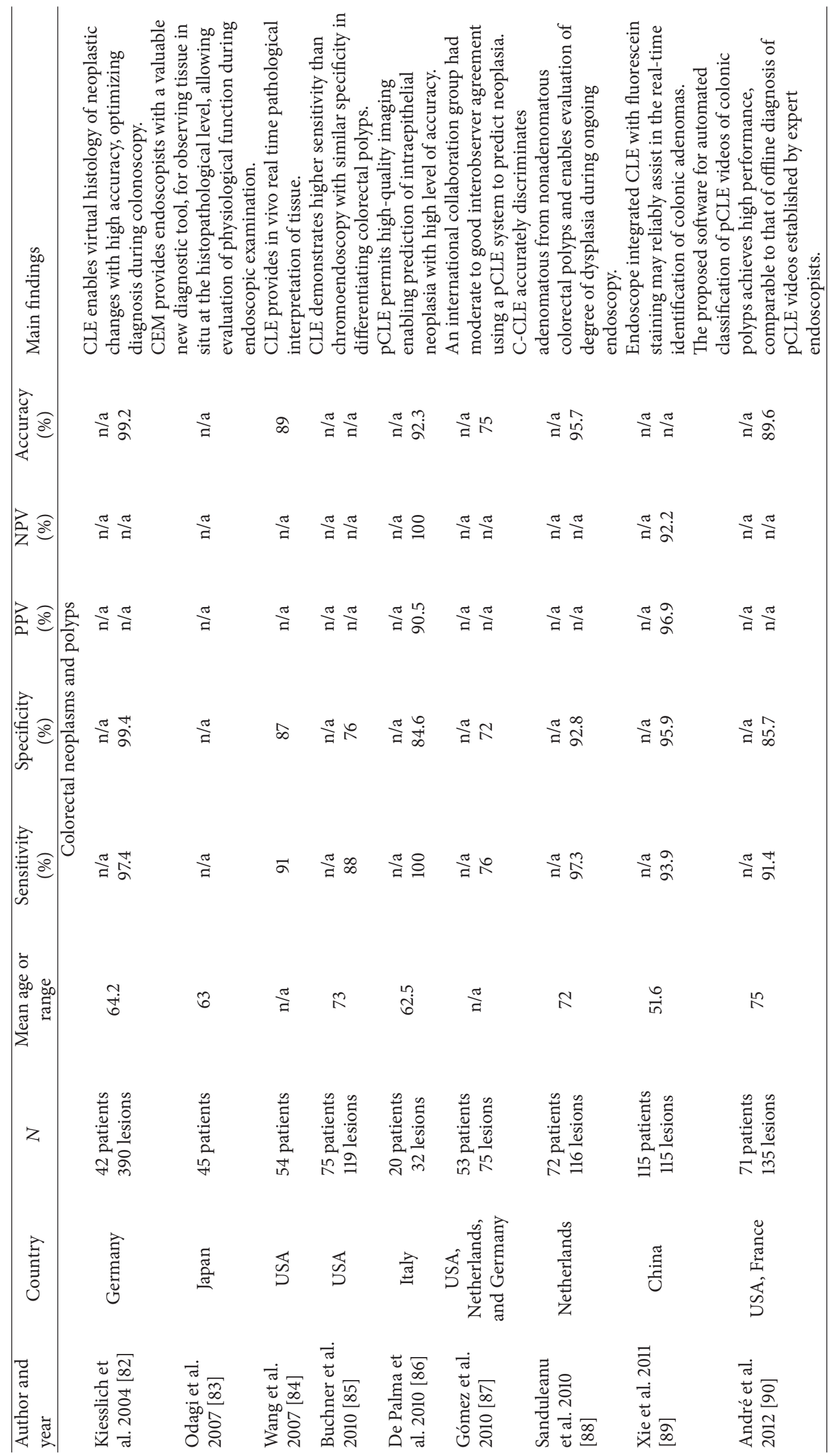




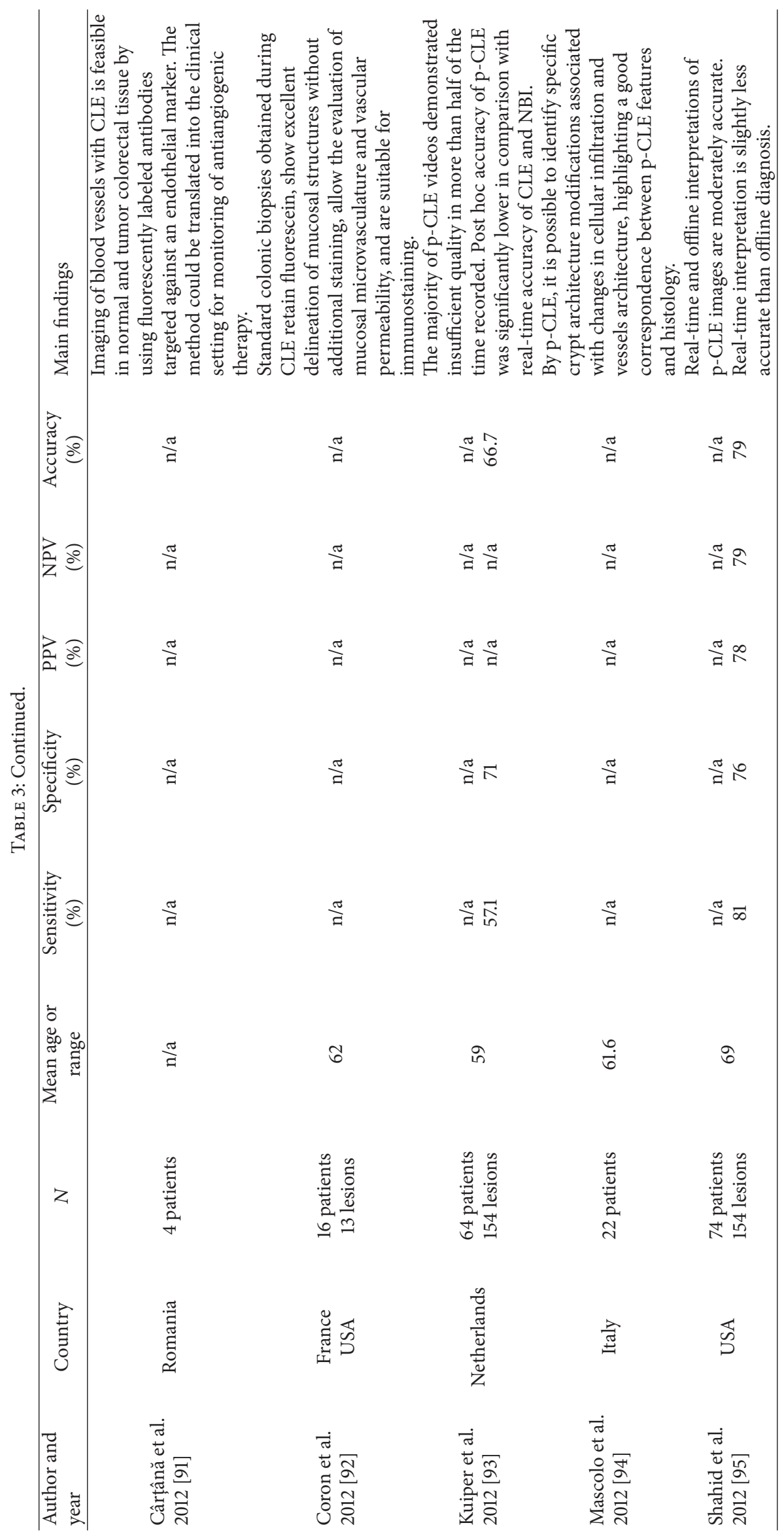




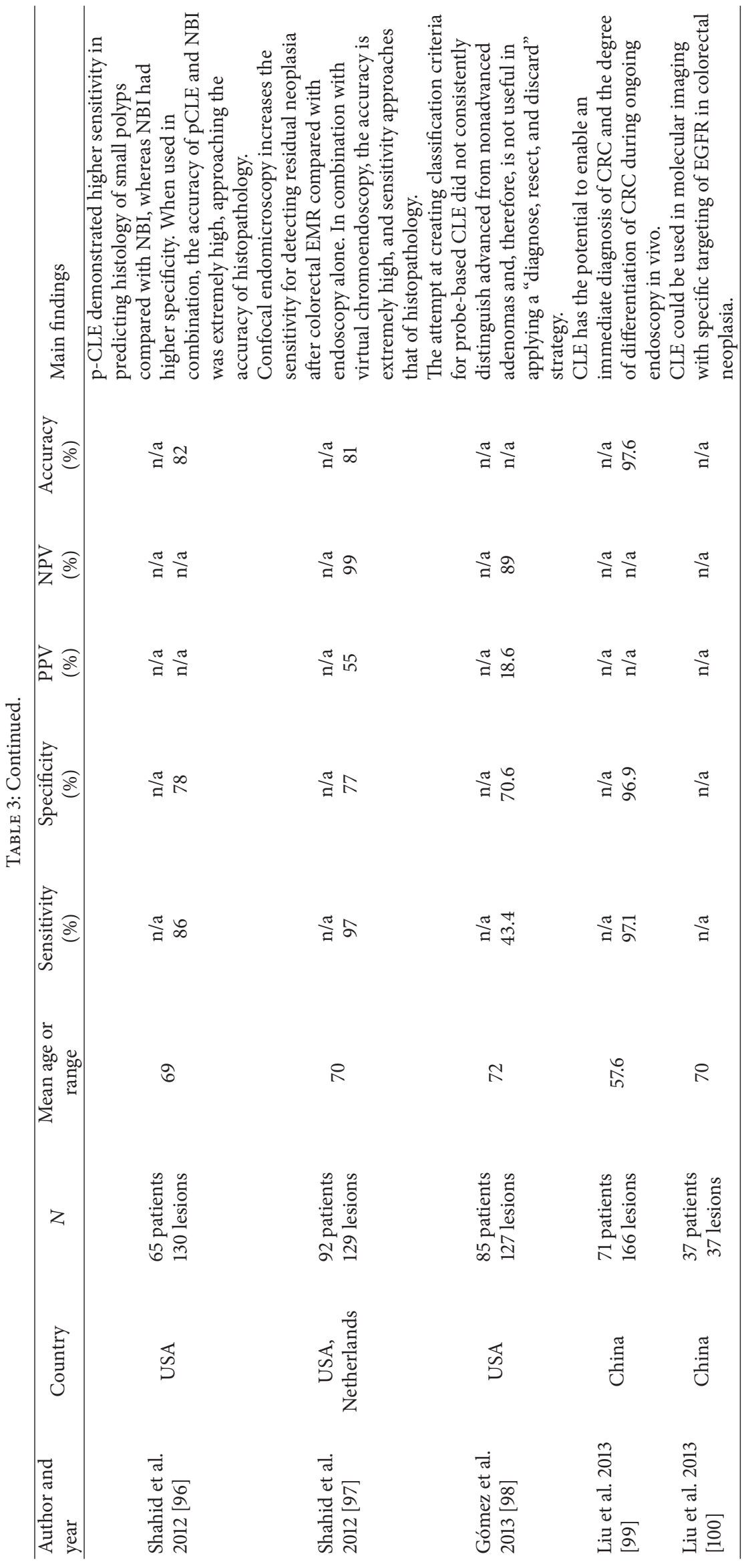




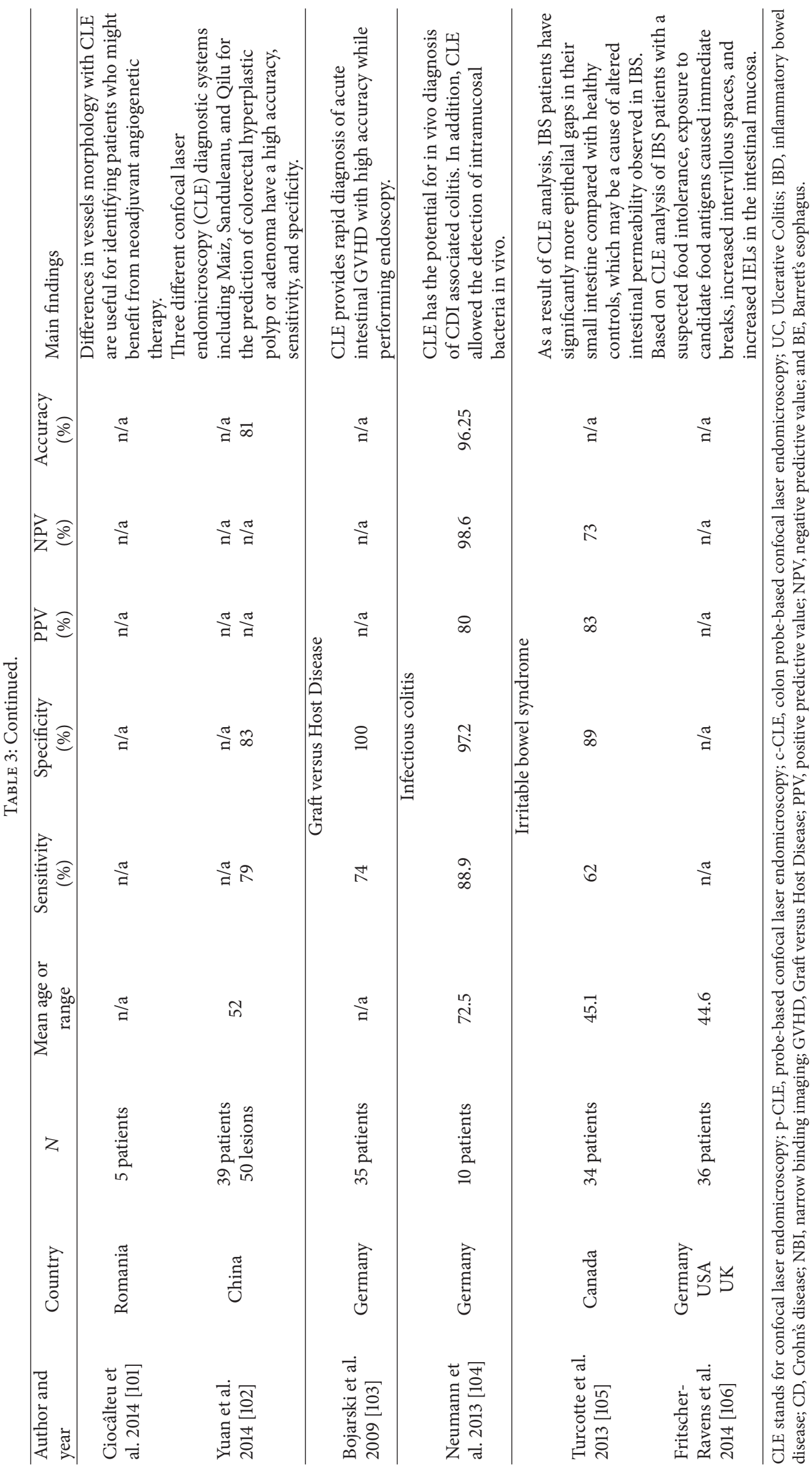




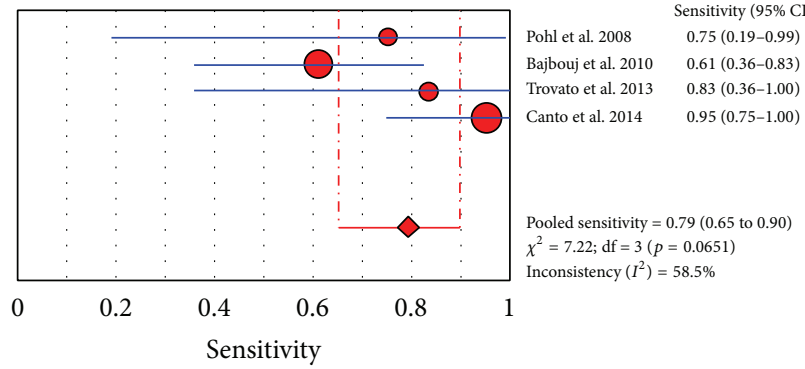

(a)

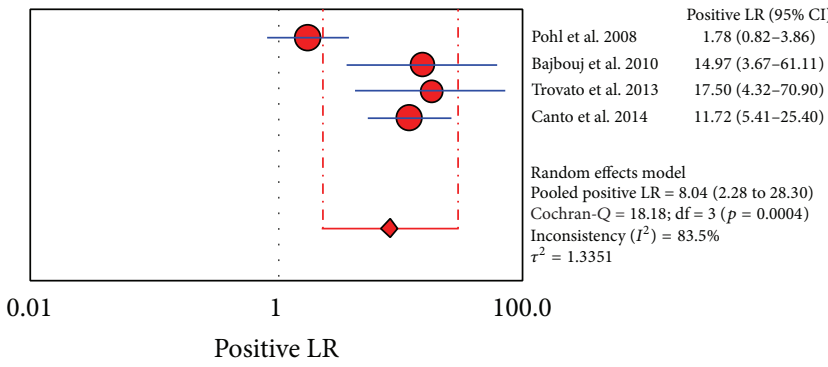

(c)

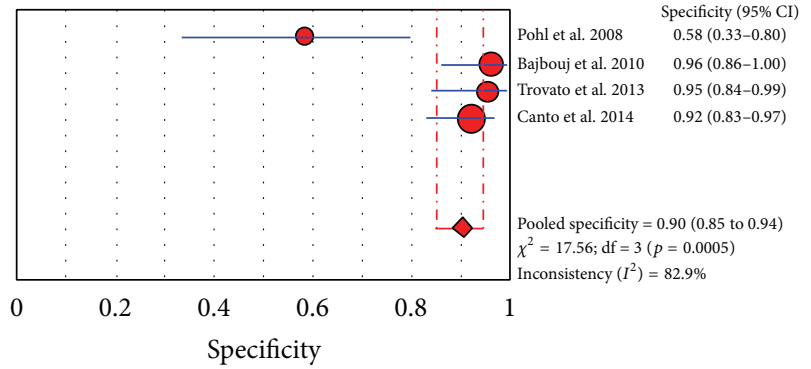

(b)

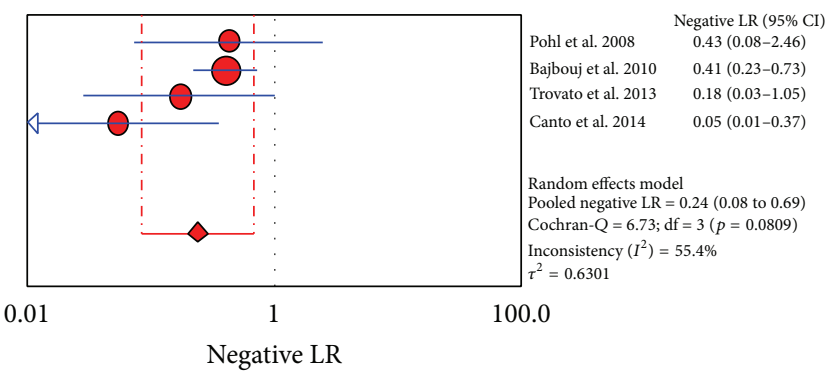

(d)

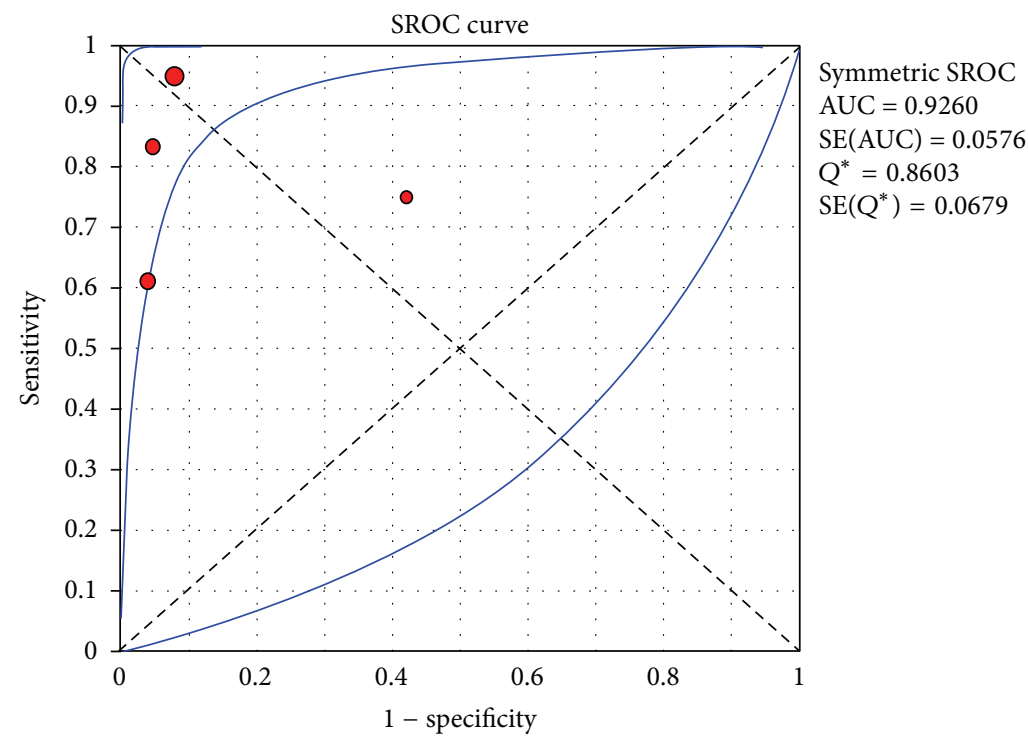

(e)

Figure 2: "Per patient" meta-analysis for CLE application in Barrett's esophagus: (a) pooled sensitivity, (b) pooled specificity, (c) pooled positive likelihood ratio (LR), (d) pooled negative LR, and (e) summary receiver operatic characteristic (SROC).

produced a pooled "per lesion" sensitivity of $83 \%\left(\mathrm{CI}_{95 \%}\right.$ : $\left.79 \%-87 \% ; I^{2}: 88.8 \%\right)$, a pooled specificity of $90 \%\left(\mathrm{CI}_{95 \%}\right.$ : $\left.87 \%-92 \% ; I^{2}: 94.8 \%\right)$, a pooled positive LR of $6.65\left(\mathrm{CI}_{95 \%}\right.$ : $\left.2.8-15.8 ; I^{2}: 90.3 \%\right)$, and a pooled negative $\mathrm{LR}$ of $0.17\left(\mathrm{CI}_{95 \%}\right.$ : $\left.0.07-0.43 ; I^{2}: 92 \%\right)$. The area under the curve was 0.9430 (Figure 6).

CLE has also been widely applied to describe IBD morphologic features, although it was not possible to perform a quantitative analysis of this application. In this particular indication, CLE appears to be a safe and feasible diagnostic tool for imaging bowel morphology, assessing disease activity, and predicting therapeutic responses.
3.5. Biliary Duct. In biliary tract and pancreatic cysts, routine forceps are not accurate for sampling and pathology exam fails to address diagnosis. CLE with in situ diagnosis might be a valuable alternative. However, few studies have been carefully conducted.

CLE has been applied for the diagnosis of common biliary duct lesions and to distinguish between benign and malignant strictures in patients with indeterminate biliary stenosis [2, 107-115] (Table 4). These applications were prospectively evaluated in several studies; of these, we performed a meta-analysis of 8 studies [107-109, 111-115], which resulted in a pooled sensitivity of $90 \%\left(\mathrm{CI}_{95 \%}: 86 \%-94 \% ; I^{2}: 1.6 \%\right)$, 


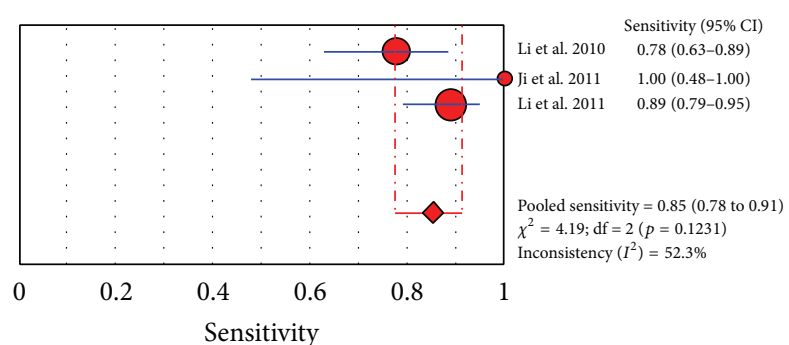

(a)

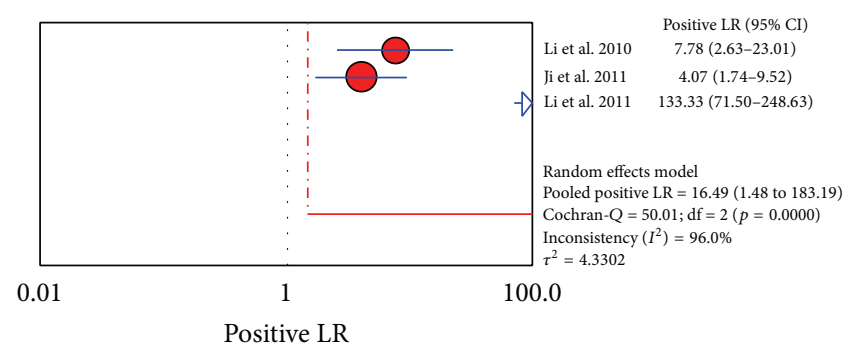

(c)

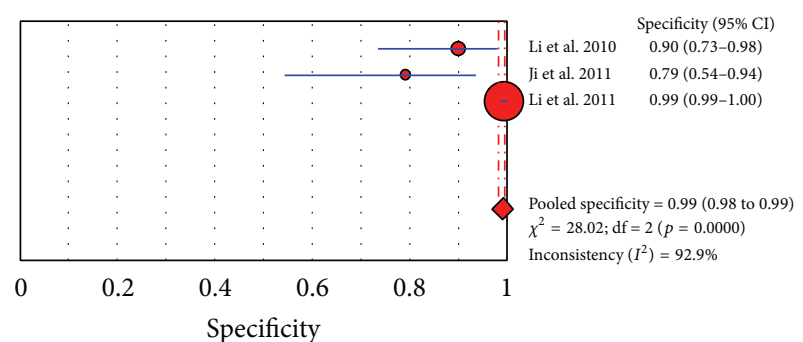

(b)

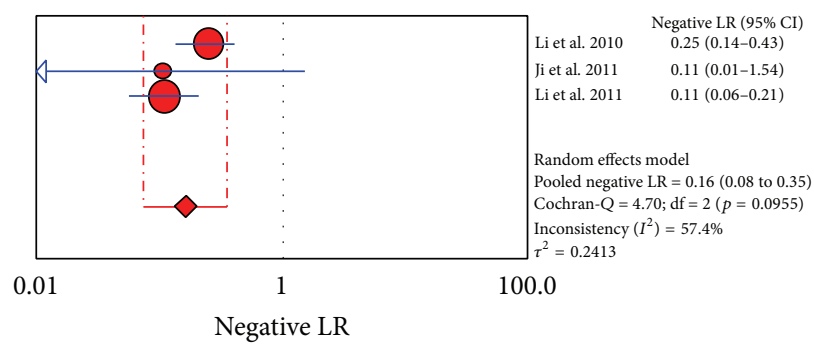

(d)

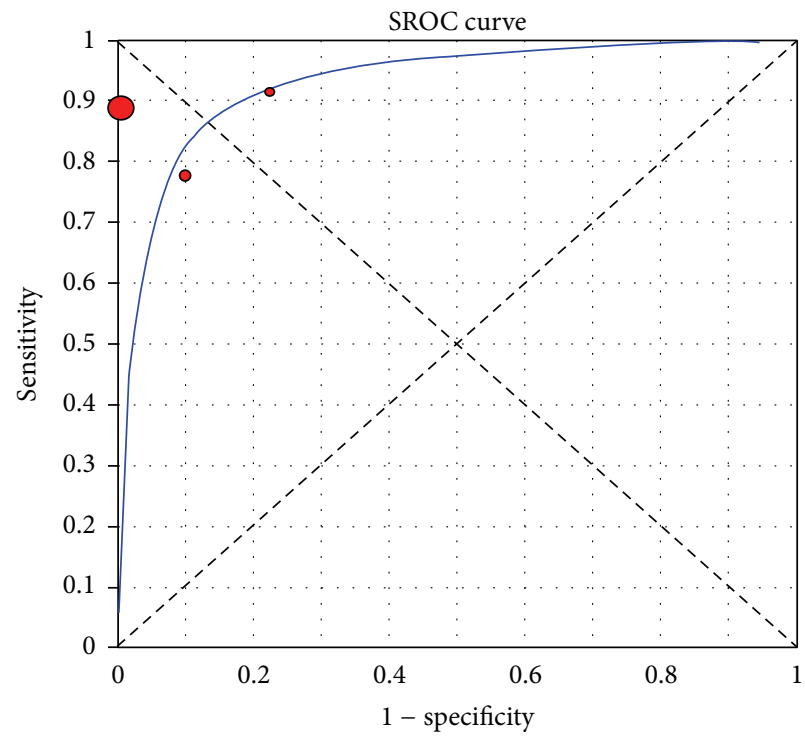

Symmetric SROC

AUC $=0.9296$

$\mathrm{SE}(\mathrm{AUC})=0.0606$

$Q^{*}=0.8647$

$\operatorname{SE}\left(Q^{*}\right)=0.0727$

(e)

FIGURE 3: "Per patient" meta-analysis for the application of CLE in detection and diagnosis of neoplastic lesions in the stomach: (a) pooled sensitivity, (b) pooled specificity, (c) pooled positive likelihood ratio (LR), (d) pooled negative LR, and (e) summary receiver operatic characteristic (SROC) curve.

a pooled specificity of $72 \%\left(\mathrm{CI}_{95 \%}: 65 \%-79 \% ; I^{2}: 0 \%\right)$, a pooled positive LR of $3.21\left(\mathrm{CI}_{95 \%}: 2.55-4.11 ; I^{2}: 0 \%\right)$, and a pooled negative LR of $0.15\left(\mathrm{CI}_{95 \%}: 0.10-0.23 ; I^{2}: 0 \%\right)$. The area under the curve was 0.8578 (Figure 7).

3.6. Pancreas. Currently, in humans, the use of nCLE is exclusively applied to pancreatic tissue. The literature concerning this technique includes a small number of studies that examined the characterization of pancreatic lesions, such as pancreatic cystic neoplasms $[14,15,117]$, indeterminate pancreatic duct strictures [116], and serous cystadenomas [118] (Table 5). A meta-analysis of two studies $[14,117]$ that applied nCLE for the diagnosis of pancreatic cyst neoplasms produced a pooled sensitivity of $68 \%\left(\mathrm{CI}_{95 \%}: 55 \%-80 \% ; I^{2}: 79.8 \%\right)$, a pooled specificity of $90 \%\left(\mathrm{CI}_{95 \%}: 74 \%-98 \% ; I^{2}: 82.4 \%\right)$, a pooled positive LR of $6.72\left(\mathrm{CI}_{95 \%}: 0.94-47.89 ; I^{2}: 52 \%\right)$, and a pooled negative LR of $0.30\left(\mathrm{CI}_{95 \%}: 0.10-0.84 ; I^{2}: 60.6 \%\right)$ (Figure $\mathrm{S} 3$ ).

3.7. Study Quality Assessment. Based on the GRADE system, the overall quality of the evidence included in our analysis was judged as low (10 studies had a very low level of quality; 59 were low; 31 were moderate; and 2 were high). The risk of bias in the comparative randomized and nonrandomized studies was evaluated by two independent reviewers (Alessandro 


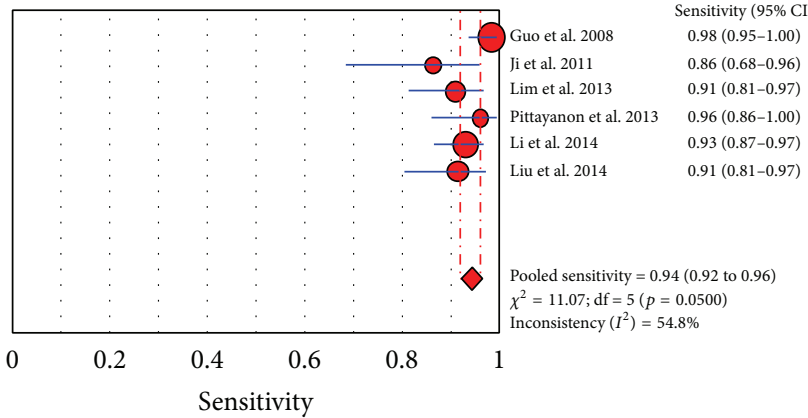

(a)

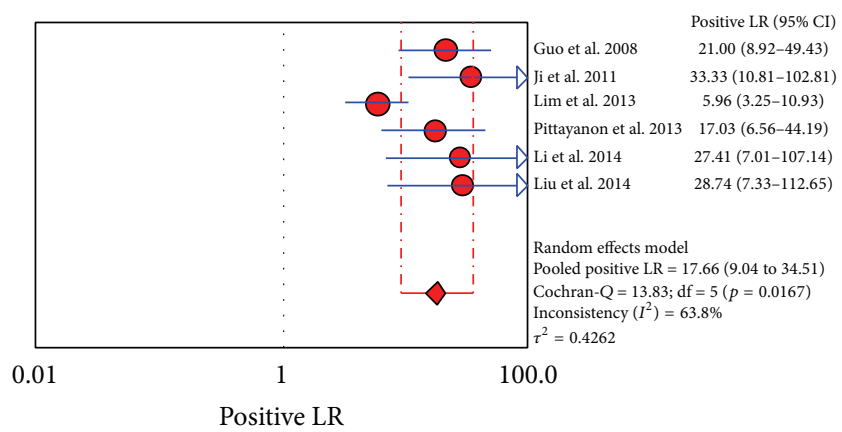

(c)

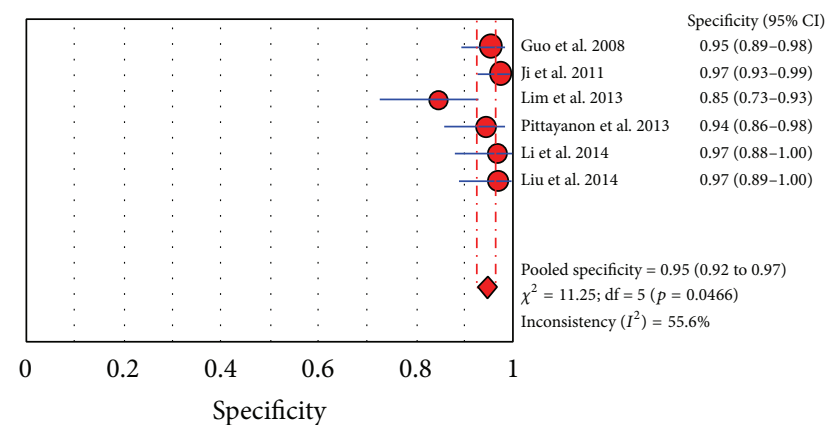

(b)

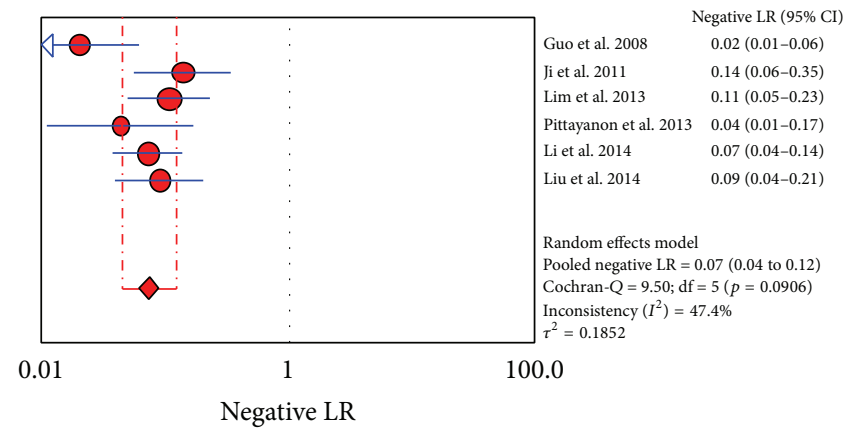

(d)

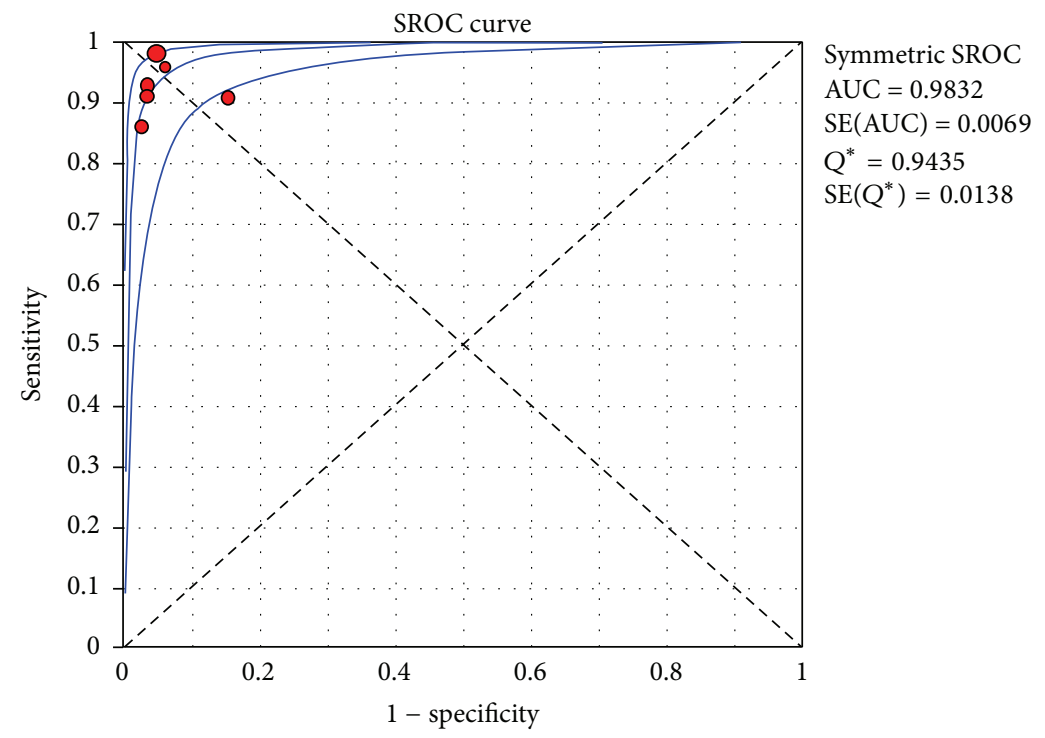

(e)

Figure 4: "Per biopsy" meta-analysis for the application of CLE in gastritis and gastric metaplasia: (a) pooled sensitivity, (b) pooled specificity, (c) pooled positive likelihood ratio (LR), (d) pooled negative LR, and (e) summary receiver operatic characteristic (SROC) curve.

Fugazza, Federica Gaiani) based on Cochrane and NOS criteria. Specifically, two RCTs $[55,77]$ were classified as having a low risk of bias, and 6 RCTs had a high risk of bias [22, 25, $27,28,31,54]$ (Table S2). Based on NOS criteria, 5 studies [29, $52,62,70,74]$ were classified as having a low risk of bias, and $17[36,39,48,59-61,63,66-69,71,72,78,94,106,112]$ had a high risk of bias (Table S3).

\section{Discussion}

To the best of our knowledge, this is the first systematic review to analyze the diagnostic accuracy of CLE across all of the applications for which it has been used.

A crucial difference between CLE and alternative endoscopic techniques is that CLE does not merely identify a lesion, 


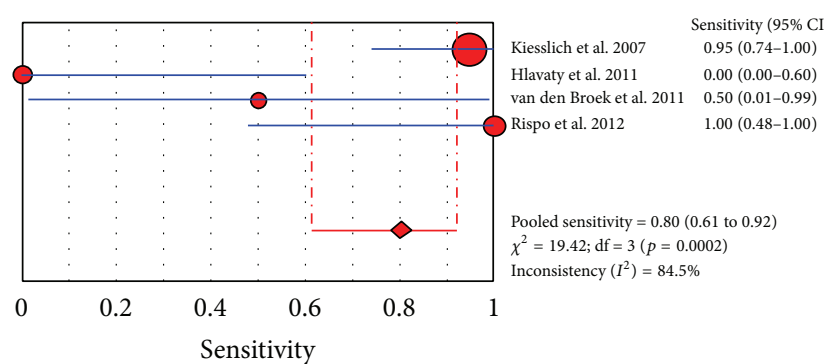

(a)

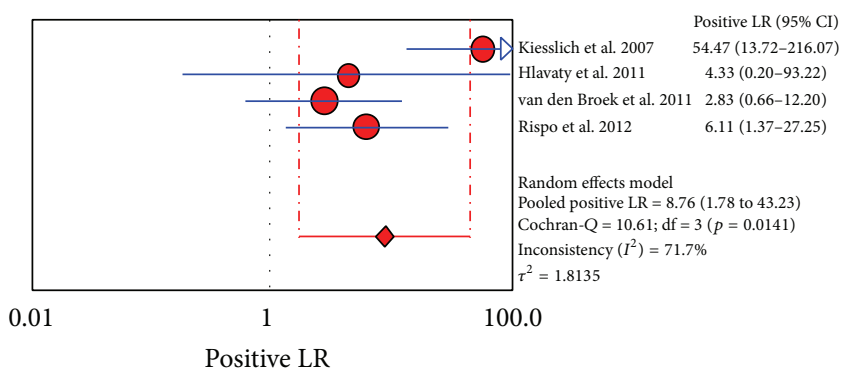

(c)

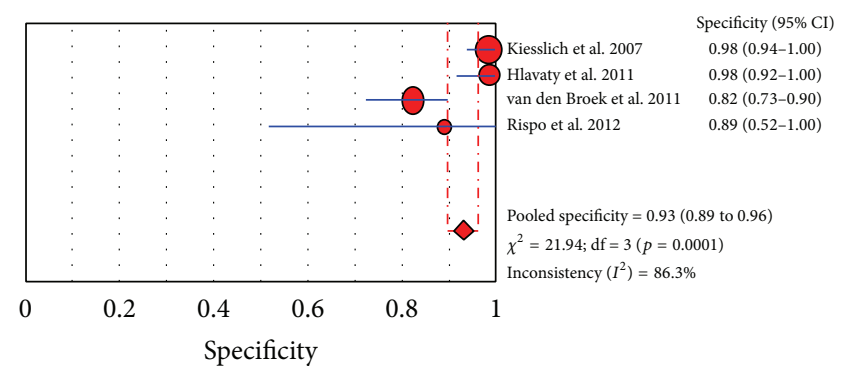

(b)

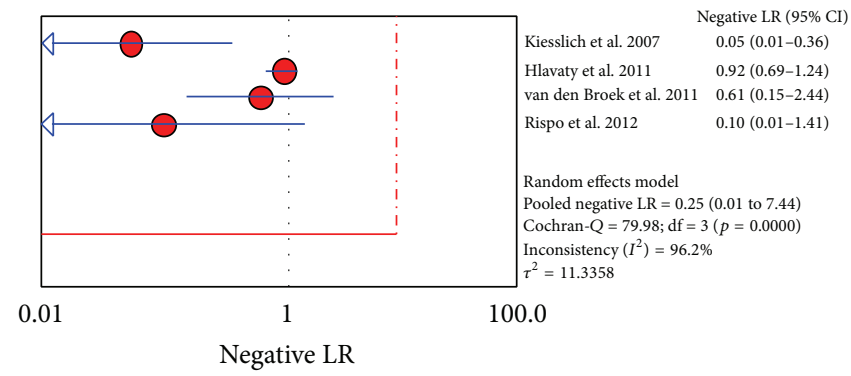

(d)

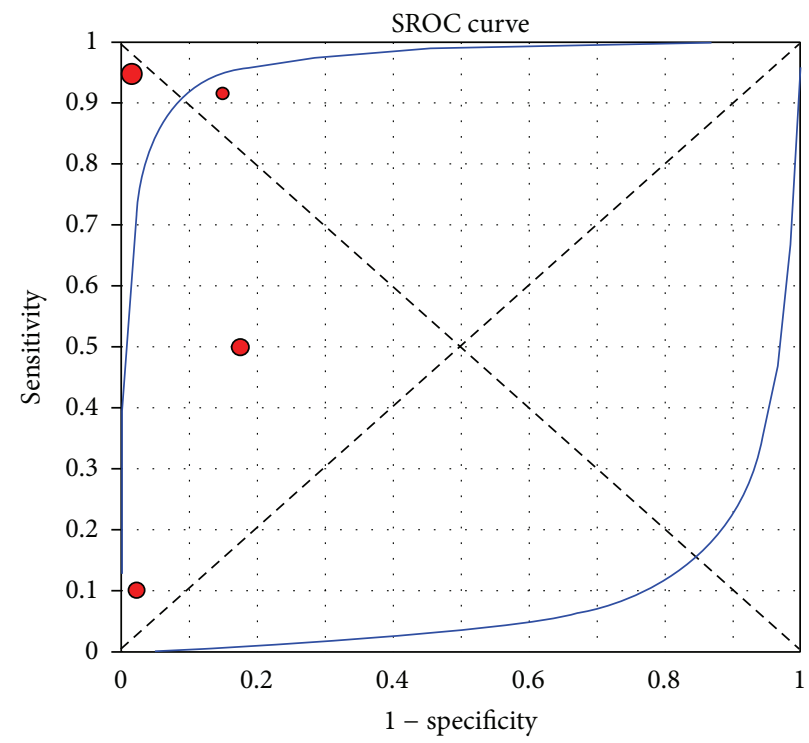

Symmetric SROC AUC $=0.9630$ $\mathrm{SE}(\mathrm{AUC})=0.0512$ $Q^{*}=0.9090$ $\mathrm{SE}\left(Q^{*}\right)=0.0768$

(e)

FIGURE 5: "Per lesion" meta-analysis for the application of CLE in the detection of dysplasia and neoplasia in inflammatory bowel disease patients: (a) pooled sensitivity, (b) pooled specificity, (c) pooled positive likelihood ratio (LR), (d) pooled negative LR, and (e) summary receiver operatic characteristic (SROC) curve.

but it also enables the discrimination of benign or malignant features via direct and immediate microscopic investigation, which can be performed simultaneously with endoscopic examination [119]. Despite this, CLE is still viewed as an expensive tool that requires standardized criteria for the diagnosis of select pathologies and needs specific training to interpret CLE images before it can be implemented as a routine diagnostic tool.

4.1. CLE in the Esophagus. Several studies have validated the diagnostic and therapeutic role of CLE with respect to premalignant lesions and cancers of the upper GI tract. In a feasibility study, the use of pCLE demonstrated an up to $92 \%$ accuracy rate in detecting malignant and premalignant modifications of GI mucosa compared to conventional histopathology [120]. However, the primary application of CLE in esophageal tissue has been for the detection of high-grade dysplasia and cost-effective treatment strategies of BE. In particular, CLE has been used for follow-up of BE patients presenting with HG dysplasia and to define the lateral extent of neoplasias prior to therapy.

It has been demonstrated that when using endomicroscopy for the surveillance of $\mathrm{BE}$, the number of required biopsies can be significantly decreased by up to $87 \%$ [22], as this 


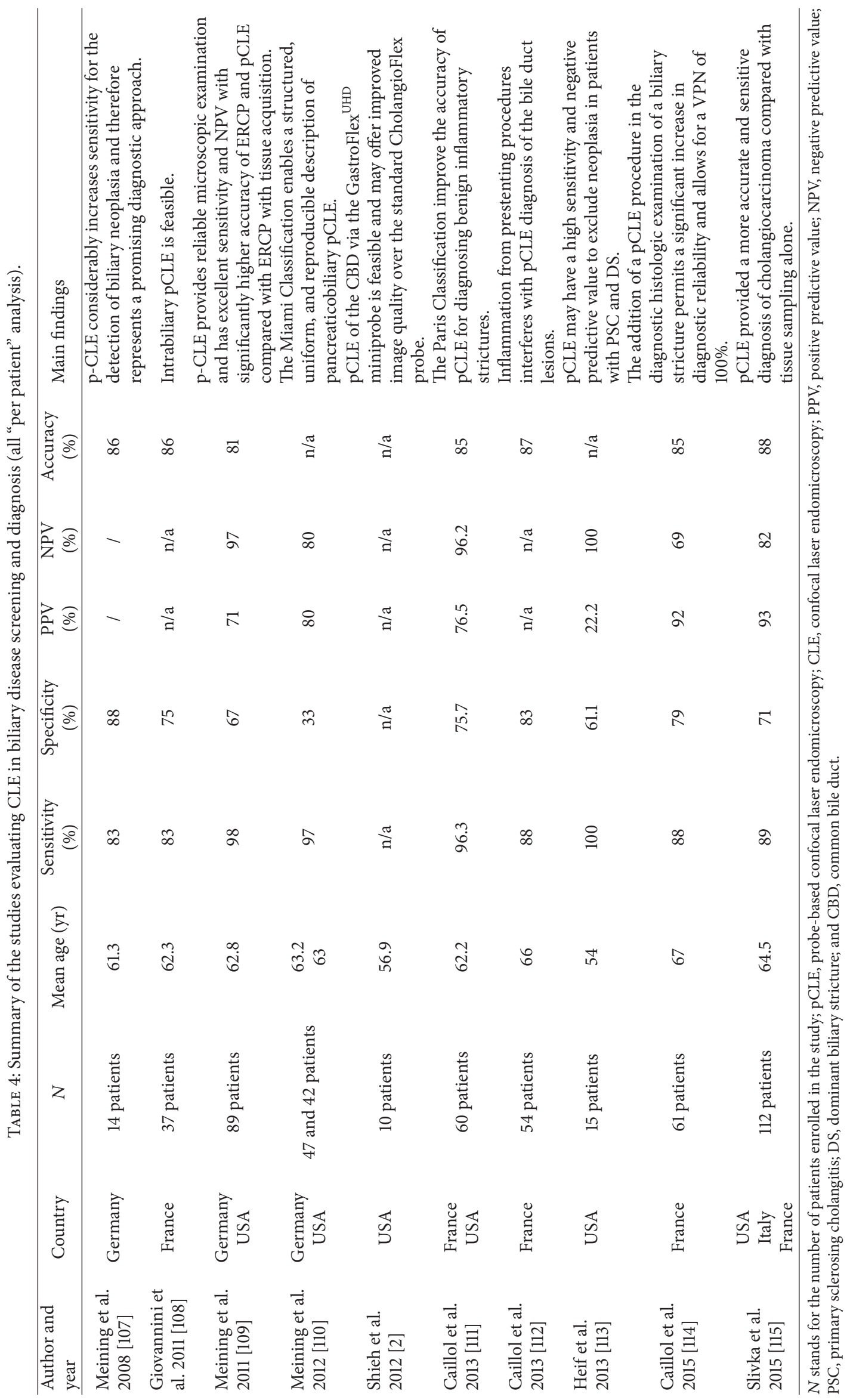




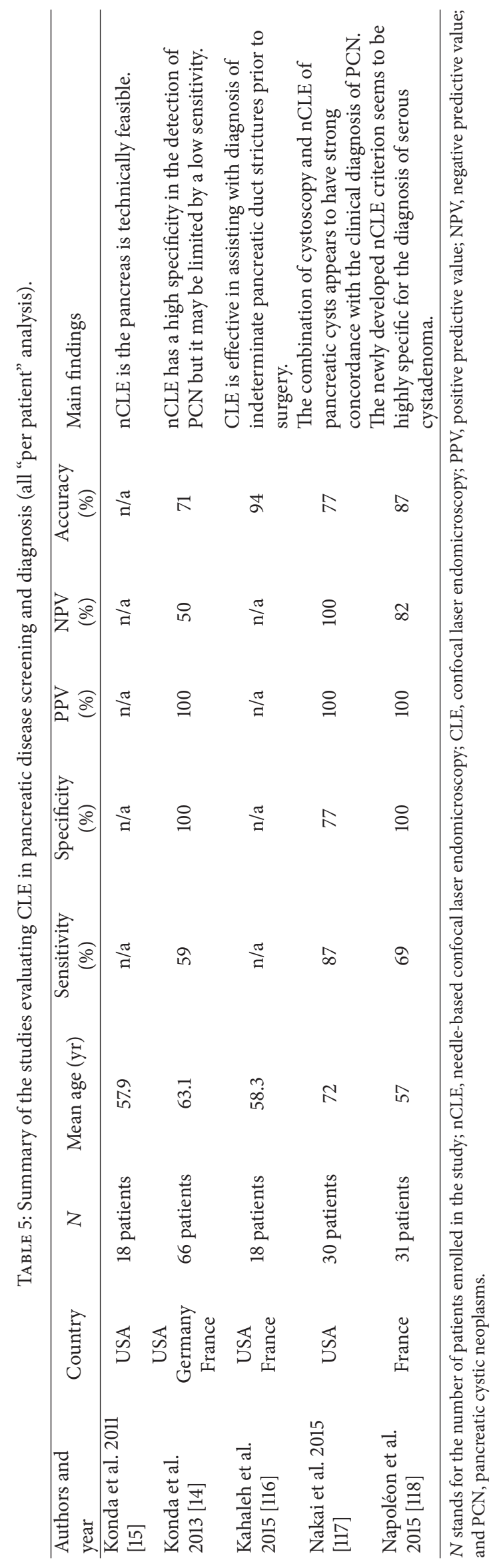




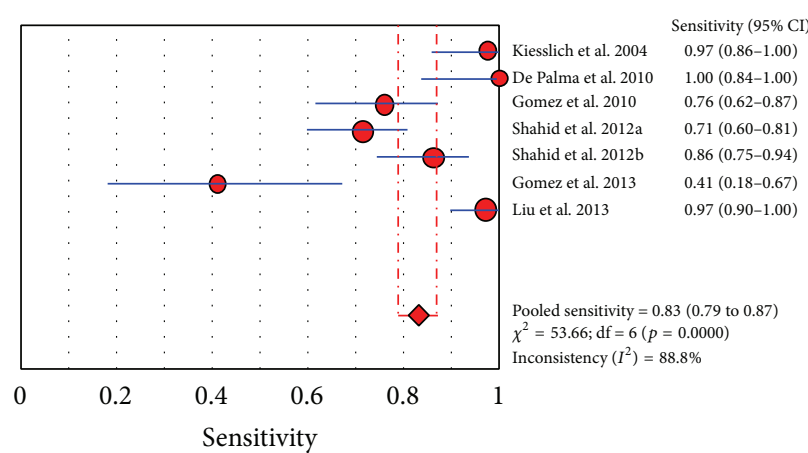

(a)

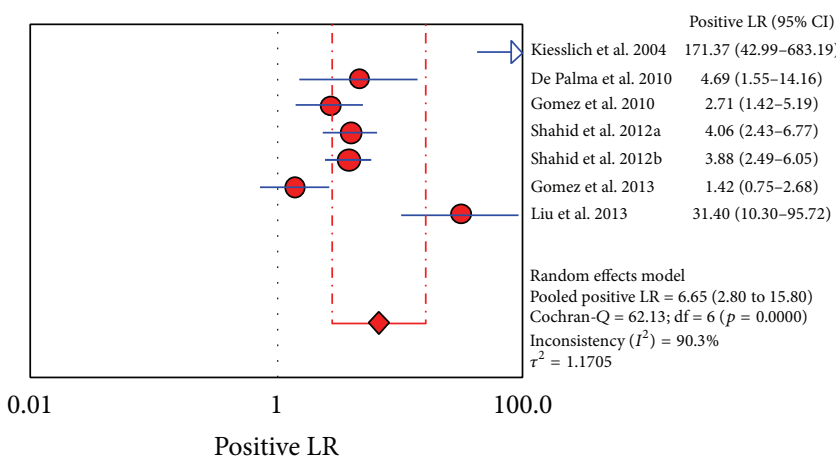

(c)

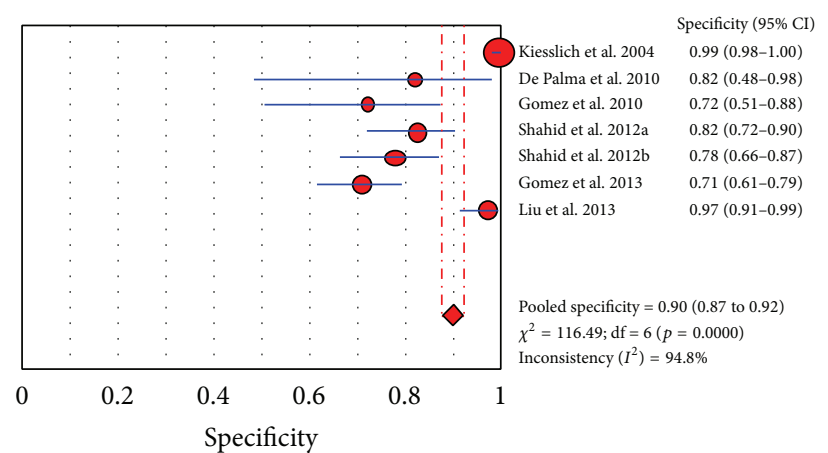

(b)

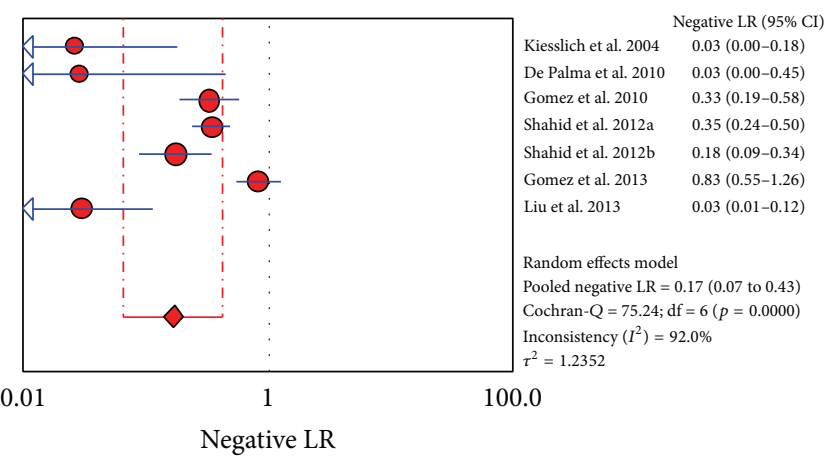

(d)

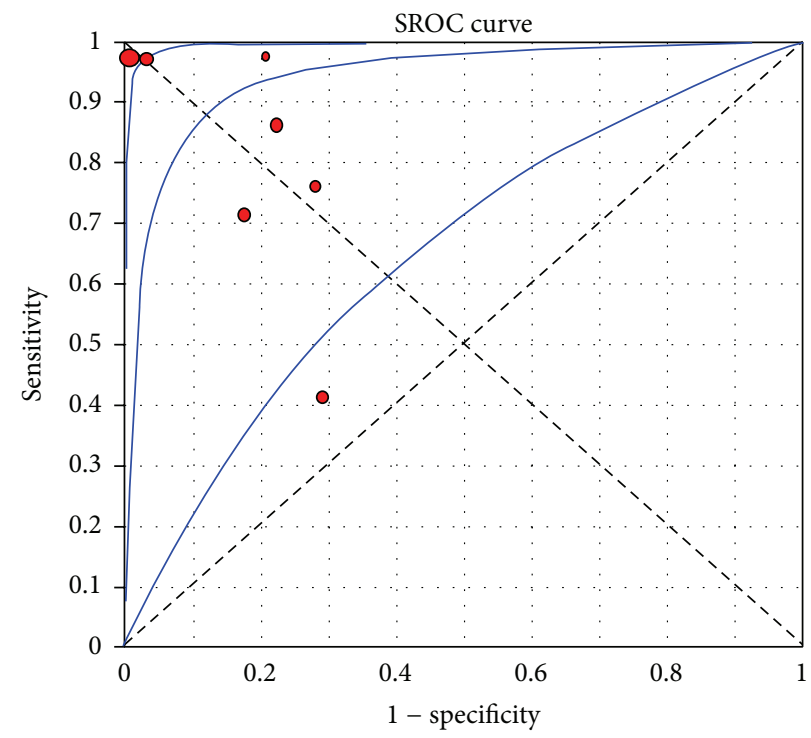

Symmetric SROC

$\mathrm{AUC}=0.9430$

$\mathrm{SE}(\mathrm{AUC})=0.0487$

$Q^{*}=0.8812$

$\mathrm{SE}\left(Q^{*}\right)=0.0628$

(e)

FIGURE 6: "Per lesion" meta-analysis for the application of CLE in colorectal neoplasms and malignant foci in polypoid lesions: (a) pooled sensitivity, (b) pooled specificity, (c) pooled positive likelihood ratio (LR), (d) pooled negative LR, and (e) summary receiver operatic characteristic (SROC) curve.

technique produces a higher diagnostic yield for the detection of neoplasia compared to random biopsy. This improved yield directly results from the need to sample only the suspicious areas that have been identified by CLE $[22,31]$. However, what the optimal surveillance biopsy procedure is in BE patients remains unclear. Current surveillance programs, such as the four-quadrant Seattle biopsy protocol, are relatively expensive and time-consuming [29]. The Preservation and Incorporation of Valuable Endoscopic Innovation (PIVI) initiative that was recently implemented by the American Society of Gastrointestinal Endoscopy (ASGE) [121] recommends that, before replacing the current Seattle protocol, a targeted 


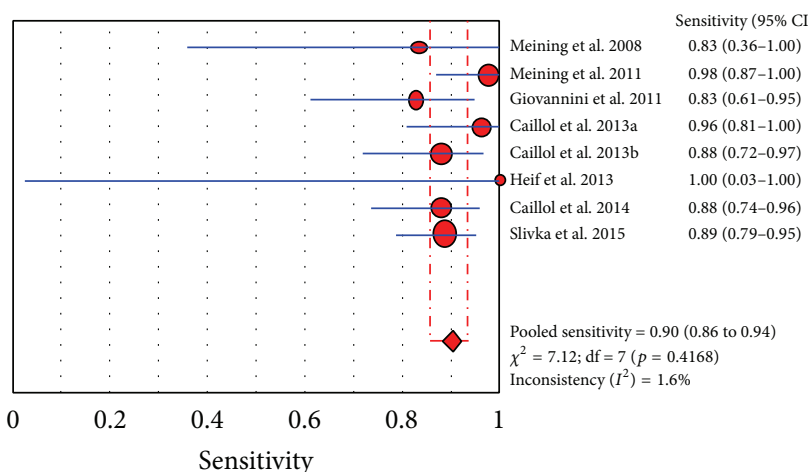

(a)

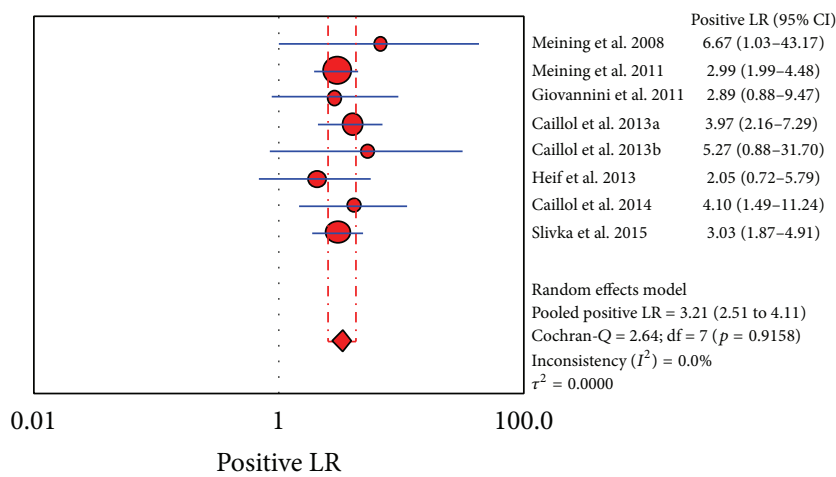

(c)

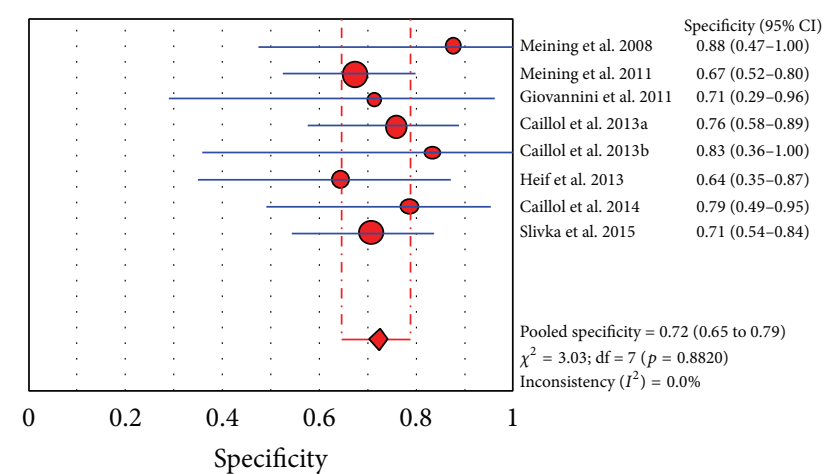

(b)

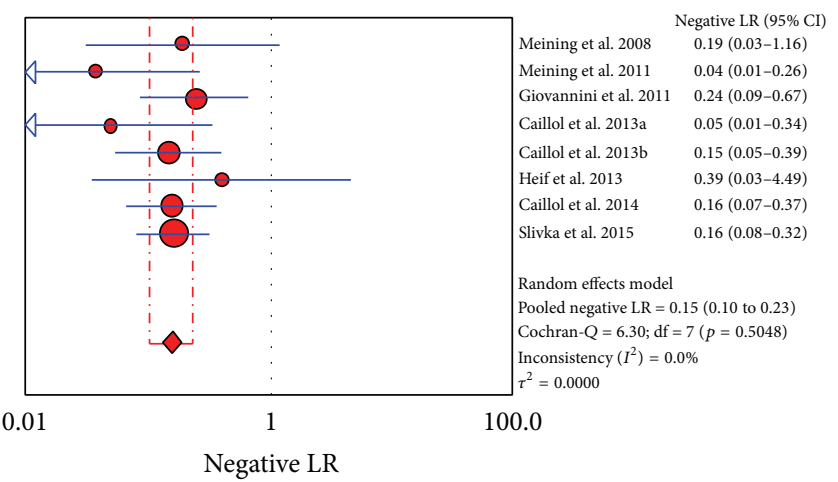

(d)

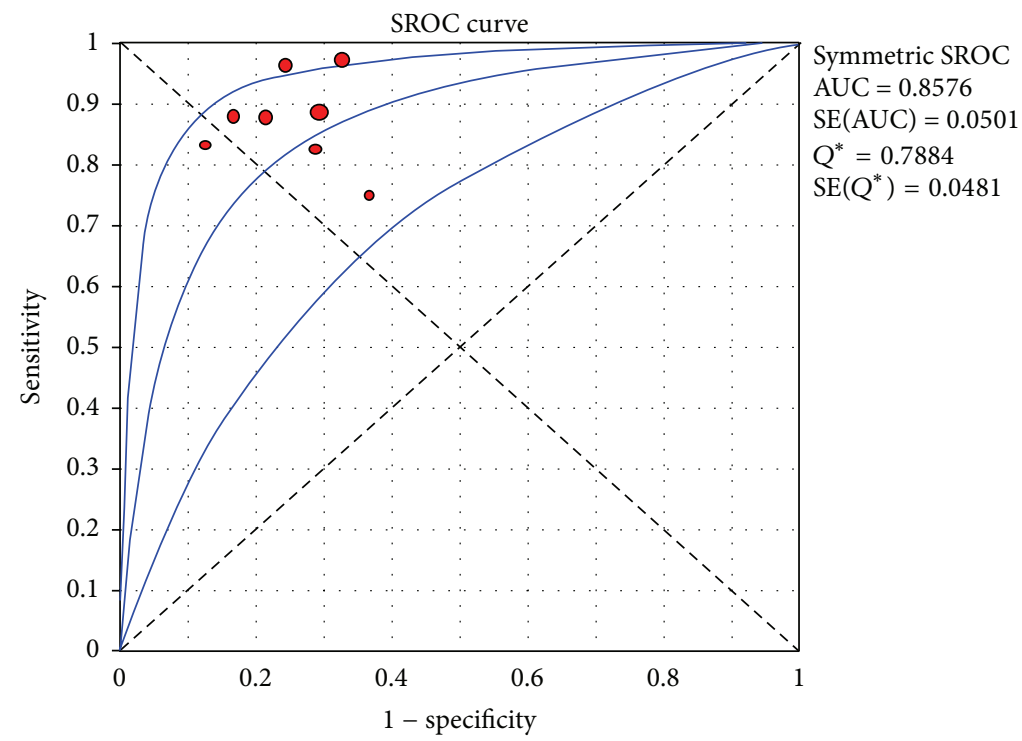

(e)

FIGURE 7: "Per patient" meta-analysis for biliary duct application of CLE: (a) pooled sensitivity, (b) pooled specificity, (c) pooled positive likelihood ratio (LR), (d) pooled negative LR, and (e) summary receiver operatic characteristic (SROC) curve.

imaging technique should have a per patient sensitivity of at least $90 \%$, an NPV of at least $98 \%$, and a specificity of at least $80 \%$ in the detection of high-grade dysplasia or early adenocarcinoma. The present meta-analysis demonstrated that CLE yields a "per biopsy" pooled sensitivity of $58 \%$ and a pooled specificity of $90 \%$, which was slightly increased to $79 \%$ sensitivity in the "per patient" analysis. Therefore, based on the PIVI initiative requirements, using CLE for the surveillance of $\mathrm{BE}$ does not appear to be sensitive enough to replace the Seattle biopsy protocol. However, a recent multicenter RCT showed that combining CLE with highdefinition WLE surpassed the PIVI threshold, with a per 
patient sensitivity of $95 \%$, an NPV of $98 \%$ and a specificity of $92 \%[31,122]$. Thus, the combined use of CLE with highdefinition WLE or NBI may be considered a valuable diagnostic tool for premalignant and malignant lesions. Nevertheless, prospective medicoeconomic studies have yet to be conducted.

4.2. CLE in the Stomach and Duodenum. CLE has been used for the description and detection of several gastric diseases, including polyps, metaplasia, and neoplastic lesions, as well as for the surveillance of gastric resections, Helicobacter pylorirelated gastritis, and celiac disease. However, knowledge surrounding the application of CLE to the stomach remains limited (although promising), and further clinical trials are needed to support the clinical impact of employing CLE in the diagnosis and management of total gastric atrophy.

The usefulness of CLE is especially evident with regard to targeting biopsies of specific pathological mucosal areas. Indeed, a recent prospective study comparing NBI, chromoendoscopy (CE), and CLE for the diagnosis of atrophic gastritis found NBI to be equivalent to $\mathrm{CE}$ in classifying gastric pits, whereas CLE had higher sensitivity, specificity, and accuracy than CE [53]. Furthermore, with respect to the detection of gastric intestinal metaplasia (GIM), CLE demonstrated high diagnostic yields and a substantial superiority over conventional endoscopy; with CLE, the number of biopsies needed to confirm GIM was about one-third of what was needed compared to using WLE and standard biopsies $[50,55]$ also suggesting a gain of time. This may be also the case in those patients at very high risk of early carcinoma (Lynch syndrome, $\mathrm{CDH}$ mutated individuals).

CLE has also been used for descriptive purposes; a blinded prospective study investigating gastric pit patterns provided an obvious distinction between normal mucosa, chronic inflammation, atrophy, and neoplastic mucosa and demonstrated a sensitivity and specificity for predicting gastric atrophy of $83.6 \%$ and $99.6 \%$, respectively, whereas the corresponding values for predicting gastric cancer were $90.0 \%$ and $99.4 \%$ [123].

With respect to gastric polyps and neoplastic lesions, CLE has demonstrated several promising applications, including distinguishing between adenomas and hyperplastic polyps [42], the identification of microvascular patterns [39], followup after resection [45], and the diagnosis of cancer at different stages. The majority of studies have indicated that CLE has high diagnostic yields, and elevated interobserver agreement rates were evident in most cases [42]. However, CLE accuracy can be limited by the acquisition of good quality images [38], which may not be always possible. The present meta-analysis demonstrated that CLE yields remarkable pooled sensitivity and specificity, reaching values of $85 \%$ and $99 \%$, respectively, in a "per patient" analysis.

Interestingly, CLE has also been applied to assess duodenal histology in patients with celiac disease. Employing CLE offers the prospect of diagnosing celiac disease during ongoing endoscopy and enables targeting biopsies to be performed in abnormal mucosa, thereby increasing diagnostic yield. However, although CLE appears to be sensitive and specific at detecting increased numbers of intraepithelial lymphocytes and villous atrophy, the evidence that CLE is effective for such applications remains scarce. More likely, CLE methodology would need to be improved before it could be routinely used in patients with celiac disease.

Overall, applying CLE to examine the stomach and duodenum demonstrated high sensitivity, specificity, accuracy, and positive and negative predictive values in comparison with both histopathology and other endoscopic techniques (e.g., WLE, NBI, and CE). However, these data are based on a limited number of publications and therefore caution should be used when interpreting their results.

4.3. CLE in the Colon. The increasing incidence of colorectal cancers in parallel with the increased use of colonoscopy screening necessitates continued improvements in diagnostic accuracy and precocity. Therefore, the advent of CLE has been considered to be an important and valuable innovation for the management of colorectal neoplasms and inflammatory diseases [77].

Based on the present meta-analysis, the use of CLE in the detection of colorectal neoplasms and malignant foci in polypoid lesions is associated with a pooled sensitivity of $83 \%$ and a pooled specificity of $90 \%$. This confirms the robust diagnostic power of CLE that has been observed in previous studies. Moreover, when compared to NBI, pCLE exhibited higher sensitivity ( $86 \%$ versus $64 \%$ ) and similar overall accuracy ( $82 \%$ versus $79 \%$ ), although it also exhibited lower specificity (78\% versus 92\%) [96]. Interestingly, the diagnostic accuracy of CLE does not appear to be influenced by operator expertise in the evaluation of confocal images; in contrast, learning how to perform CLE appears to involve only a short learning curve [102]. In addition to its role as a diagnostic tool, CLE is useful for evaluating the presence of residual tumor left behind following endoscopic treatment of colon polyps and does not require waiting for biopsy results. If there is an eventual need for reintervention via a complementary resection, it can therefore be carried out during a single endoscopic session [97].

CLE also demonstrated high applicability and superiority over standard endoscopy in the study of IBD. In particular, CLE can be used in the assessment of disease activity [70], in the prediction of relapse $[71,74]$, and in the description of mucosal alterations such as epithelial gaps, all of which are useful toward enhancing the comprehension of new pathogenic features that develop in patients with IBD [66]. However, the detection of neoplastic transformations in the background of chronic inflammation in IBD patients remains highly challenging. Conversely, using CLE in patients with IBD is promising approach, as it offers the possibility of directly observing microvessels by immunostaining and therefore may serve as a foundation for the development targeted antiangiogenic therapies [91, 101].

Applying CLE to patients GVHD, infectious colitis and irritable bowel syndrome has been less extensively studied; however, this technique has demonstrated good performance in these indications (100\% specificity and less invasiveness in comparison with standard diagnosis technique), although its standards remain outside of current guidelines $[103,106]$. 
4.4. CLE in the Biliary Duct. With respect to the biliary duct, the addition of CLE to histological examination results in a significant increase in diagnostic reliability [114]. Malignant pancreatobiliary strictures are difficult to diagnose, and up to $30 \%$ of patients with cholangiocarcinoma have negative sampling [111]. This is because, unlike other tumors, the majority of cholangiocarcinomas grow along bile duct walls rather than radially forming a mass [124]. Currently, biliary strictures are staged using a combination of endoscopic ultrasound and advanced imaging techniques, such as computed tomography (CT), magnetic resonance imaging (MRI), or EUS, whereas endoscopic retrograde cholangiopancreatography (ERCP) is typically used for tissue sampling, including biopsy and cytological brushing. However, the current sensitivity of each of these methods is quite low, ranging from $20 \%$ to $60 \%$ [125-127].

The present meta-analysis demonstrated that combining CLE with ERCP yields high sensitivity (90\%) in the assessment of biliary strictures. This supports the ASGE guidelines and demonstrates that CLE is a useful tool for differentiating benign from malignant biliary strictures in patients with biliary neoplasia [128].

4.5. CLE in the Pancreas. The accurate diagnosis of pancreatic cystic neoplasms continues to be problematic despite technological improvements [117]. The application of an optical needle biopsy by using nCLE may significantly improve the discrimination of mucinous and nonmucinous cysts. Based on 2 studies that were included in the meta-analysis, CLE yields a pooled sensitivity of $68 \%$ and pooled specificity of $90 \%$, which might be suboptimal and influenced by the high heterogeneity that was observed between the studies. However, the current standard diagnostic techniques, such as imaging, EUS, fluid analysis (e.g., chemistry, tumor markers), and cytology $[14,118]$, which when combined lead to a correct diagnosis in not more than $79 \%$ of cases, are also far from being satisfactory diagnostic procedures [129].

It should be noted that the literature regarding the use of nCLE for the diagnosis of pancreatic cystic neoplasms is scarce $[14,117]$ and is mainly focused on serous cystadenoma [118]. Further studies are needed to improve nCLE diagnostic power and accuracy.

4.6. Study Limitations and Future Directions. The present systematic review and meta-analysis attempted to summarize the current literature on the applications of CLE in gastrointestinal and pancreatobiliary diseases. Although a considerable number of studies were retrieved overall, the total evidence per organ is rather scarce and is often too low to draw definitive conclusions. Moreover, high heterogeneity was observed in many of our pooled data analyses, which indicates that caution is required when interpreting their results. Finally, the included studies were primarily conducted in specialized centers; thus, CLE outcomes cannot be generalized to tertiary care centers or nonspecialized institutions.

Despite these limitations, the present systematic review and meta-analysis highlights the novel and unique advantages of using CLE to provide real-time histological examination during diagnostic and therapeutic procedures. Future clinical trials should aim to improve the diagnostic accuracy of CLE in all of its possible applications, to institutionalize training programs to standardize the interpretation of CLE images, and to reduce procedure related costs and limitations to increase its application. By improving and implementing CLE techniques during routine clinical practice, we believe that in the near future CLE not only will become part of the diagnostic arsenal of the gastroenterologist/endoscopist but also may find application in related fields such as minimally invasive surgical techniques.

\section{Conclusions}

In gastrointestinal and pancreatobiliary diseases, endoscopyassociated new technologies should offer the possibility to make clear diagnosis when routine procedures make it difficultly be cost-effective with clear impact on the choice of endoscopy versus surgical therapies for macroscopic lesions and achieve early detection of malignancies in those individuals with very high risk of cancer development. CLE is one of these new technologies able to address the challenge. The overall sensitivity, specificity, accuracy, and predictive values of CLE are favorable and were often found to be superior in comparison with standard endoscopy plus histopathology. However, the widespread use of CLE remains limited by its low availability, high costs, and need for trained personnel. Moreover, there is a need for further clinical trials, including medicoeconomic evaluations, to assess the applicability and implementation of CLE in routine clinical practice, as currently very few such studies exist.

\section{Conflict of Interests}

None of the authors have a conflict of interests to disclose in relation to the present systematic review.

\section{Authors' Contribution}

Nicola de'Angelis developed the idea and designed the research study. Alessandro Fugazza, Federica Gaiani, and Maria Clotilde Carra participated in the literature review (search, evaluation, selection, and quality assessment of the articles), data extraction, data analysis, and paper drafting. Michaël Lévy, Francesco Brunetti, Fausto Catena, and Gian Luigi de'Angelis participated in the paper drafting and revisions. Nicola de'Angelis, Daniel Azoulay, and Iradj Sobhani contributed to the final version of the paper with revisions and critical review.

\section{References}

[1] S. S. Chauhan, B. K. Abu Dayyeh, Y. M. Bhat et al., "Confocal laser endomicroscopy," Gastrointestinal Endoscopy, vol. 80, no. 6, pp. 928-938, 2014.

[2] F. K. Shieh, H. Drumm, M. H. Nathanson, and P. A. Jamidar, "High-definition confocal endomicroscopy of the common bile duct," Journal of Clinical Gastroenterology, vol. 46, no. 5, pp. 401406, 2012. 
[3] T. D. Wang, "Confocal microscopy from the bench to the bedside," Gastrointestinal Endoscopy, vol. 62, no. 5, pp. 696-697, 2005.

[4] R. Kiesslich, M. Goetz, and M. F. Neurath, "Confocal laser endomicroscopy for gastrointestinal diseases," Gastrointestinal Endoscopy Clinics of North America, vol. 18, no. 3, pp. 451-466, 2008.

[5] A. L. Polglase, W. J. McLaren, S. A. Skinner, R. Kiesslich, M. F. Neurath, and P. M. Delaney, "A fluorescence confocal endomicroscope for in vivo microscopy of the upper- and the lower-GI tract," Gastrointestinal Endoscopy, vol. 62, no. 5, pp. 686-695, 2005.

[6] H. Neumann, R. Kiesslich, M. B. Wallace, and M. F. Neurath, "Confocal laser endomicroscopy: technical advances and clinical applications," Gastroenterology, vol. 139, no. 2, pp. 388.e2392.e2, 2010.

[7] G. D. De Palma, "Confocal laser endomicroscopy in the 'in vivo' histological diagnosis of the gastrointestinal tract," World Journal of Gastroenterology, vol. 15, no. 46, pp. 5770-5775, 2009.

[8] M. B. Wallace, A. Meining, M. I. Canto et al., "The safety of intravenous fluorescein for confocal laser endomicroscopy in the gastrointestinal tract," Alimentary Pharmacology and Therapeutics, vol. 31, no. 5, pp. 548-552, 2010.

[9] J. Wu, Y.-M. Pan, T.-T. Wang, and B. Hu, "Confocal laser endomicroscopy for detection of neoplasia in Barrett's esophagus: a meta-analysis," Diseases of the Esophagus, vol. 27, no. 3, pp. 248-254, 2014.

[10] M. O. Othman and M. B. Wallace, "Confocal laser endomicroscopy: is it prime time?" Journal of Clinical Gastroenterology, vol. 45, no. 3, pp. 205-206, 2011.

[11] K. Dunbar and M. Canto, "Confocal endomicroscopy," Current Opinion in Gastroenterology, vol. 24, no. 5, pp. 631-637, 2008.

[12] M. Wallace, G. Y. Lauwers, Y. Chen et al., "Miami classification for probe-based confocal laser endomicroscopy," Endoscopy, vol. 43, no. 10, pp. 882-891, 2011.

[13] Y. Nakai, H. Isayama, S. Shinoura et al., "Confocal laser endomicroscopy in gastrointestinal and pancreatobiliary diseases," Digestive Endoscopy, vol. 26, supplement 1, pp. 86-94, 2014.

[14] V. J. A. Konda, A. Meining, L. H. Jamil et al., "A pilot study of in vivo identification of pancreatic cystic neoplasms with needlebased confocal laser endomicroscopy under endosonographic guidance," Endoscopy, vol. 45, no. 12, pp. 1006-1013, 2013.

[15] V. J. A. Konda, H. R. Aslanian, M. B. Wallace, U. D. Siddiqui, J. Hart, and I. Waxman, "First assessment of needle-based confocal laser endomicroscopy during EUS-FNA procedures of the pancreas (with videos)," Gastrointestinal Endoscopy, vol. 74, no. 5, pp. 1049-1060, 2011.

[16] V. Becker, M. B. Wallace, P. Fockens et al., "Needle-based confocal endomicroscopy for in vivo histology of intra-abdominal organs: first results in a porcine model (with videos)," Gastrointestinal Endoscopy, vol. 71, no. 7, pp. 1260-1266, 2010.

[17] J. P. Higgins, D. G. Altman, P. C. Gøtzsche et al., "The Cochrane Collaboration's tool for assessing risk of bias in randomised trials," British Medical Journal, vol. 343, Article ID d5928, 2011.

[18] A. Stang, "Critical evaluation of the Newcastle-Ottawa scale for the assessment of the quality of nonrandomized studies in metaanalyses," European Journal of Epidemiology, vol. 25, no. 9, pp. 603-605, 2010.

[19] J. Zamora, V. Abraira, A. Muriel, K. Khan, and A. Coomarasamy, "Meta-DiSc: a software for meta-analysis of test accuracy data," BMC Medical Research Methodology, vol. 6, article 31, 2006.
[20] R. Kiesslich, L. Gossner, M. Goetz et al., "In vivo histology of Barrett's esophagus and associated neoplasia by confocal laser endomicroscopy," Clinical Gastroenterology and Hepatology, vol. 4, no. 8, pp. 979-987, 2006.

[21] H. Pohl, T. Rösch, M. Vieth et al., "Miniprobe confocal laser microscopy for the detection of invisible neoplasia in patients with Barrett's oesophagus," Gut, vol. 57, no. 12, pp. 1648-1653, 2008.

[22] K. B. Dunbar, P. Okolo III, E. Montgomery, and M. I. Canto, "Confocal laser endomicroscopy in Barrett's esophagus and endoscopically inapparent Barrett's neoplasia: a prospective, randomized, double-blind, controlled, crossover trial," Gastrointestinal Endoscopy, vol. 70, no. 4, pp. 645-654, 2009.

[23] M. B. Wallace, P. Sharma, C. Lightdale et al., "Preliminary accuracy and interobserver agreement for the detection of intraepithelial neoplasia in Barrett's esophagus with probe-based confocal laser endomicroscopy," Gastrointestinal Endoscopy, vol. 72, no. 1, pp. 19-24, 2010.

[24] M. Bajbouj, M. Vieth, T. Rösch et al., "Probe-based confocal laser endomicroscopy compared with standard four-quadrant biopsy for evaluation of neoplasia in Barrett's esophagus," Endoscopy, vol. 42, no. 6, pp. 435-440, 2010.

[25] P. Sharma, A. R. Meining, E. Coron et al., "Real-time increased detection of neoplastic tissue in Barrett's esophagus with probebased confocal laser endomicroscopy: final results of an international multicenter, prospective, randomized, controlled trial," Gastrointestinal Endoscopy, vol. 74, no. 3, pp. 465-472, 2011.

[26] C. Jayasekera, A. C. F. Taylor, P. V. Desmond, F. Macrae, and R. Williams, "Added value of narrow band imaging and confocal laser endomicroscopy in detecting Barrett's esophagus neoplasia," Endoscopy, vol. 44, no. 12, pp. 1089-1095, 2012.

[27] M. B. Wallace, J. E. Crook, M. Saunders et al., "Multicenter, randomized, controlled trial of confocal laser endomicroscopy assessment of residual metaplasia after mucosal ablation or resection of GI neoplasia in Barrett's esophagus," Gastrointestinal Endoscopy, vol. 76, no. 3, pp. 539.el-547.el, 2012.

[28] V. X. Nguyen, C. C. Nguyen, G. De Petris, V. K. Sharma, and A. Das, "Confocal endomicroscopy (CEM) improves efficiency of Barrett surveillance," Journal of Interventional Gastroenterology, vol. 2, no. 2, pp. 61-65, 2012.

[29] H. Bertani, M. Frazzoni, E. Dabizzi et al., "Improved detection of incident dysplasia by probe-based confocal laser endomicroscopy in a Barrett's esophagus surveillance program," Digestive Diseases and Sciences, vol. 58, no. 1, pp. 188-193, 2013.

[30] C. Trovato, A. Sonzogni, D. Ravizza et al., "Confocal laser endomicroscopy for in vivo diagnosis of Barrett's oesophagus and associated neoplasia: a pilot study conducted in a single Italian centre," Digestive and Liver Disease, vol. 45, no. 5, pp. 396-402, 2013.

[31] M. I. Canto, S. Anandasabapathy, W. Brugge et al., "In vivo endomicroscopy improves detection of Barrett's esophagusrelated neoplasia: a multicenter international randomized controlled trial (with video)," Gastrointestinal Endoscopy, vol. 79, no. 2, pp. 211-221, 2014.

[32] O. Pech, T. Rabenstein, H. Manner et al., "Confocal laser endomicroscopy for in vivo diagnosis of early squamous cell carcinoma in the esophagus," Clinical Gastroenterology and Hepatology, vol. 6, no. 1, pp. 89-94, 2008.

[33] H. Liu, Y. Q. Li, T. Yu et al., "Confocal laser endomicroscopy for superficial esophageal squamous cell carcinoma," Endoscopy, vol. 41, no. 2, pp. 99-106, 2009. 
[34] Y. Iguchi, Y. Niwa, R. Miyahara et al., "Pilot study on confocal endomicroscopy for determination of the depth of squamous cell esophageal cancer in vivo," Journal of Gastroenterology and Hepatology, vol. 24, no. 11, pp. 1733-1739, 2009.

[35] K. Venkatesh, M. Cohen, A. Abou-Taleb, S. Thomas, C. Taylor, and M. Thomson, "A new method in the diagnosis of reflux esophagitis: confocal laser endomicroscopy," Gastrointestinal Endoscopy, vol. 75, no. 4, pp. 864-869, 2012.

[36] C.-L. Chu, Y.-B. Zhen, G.-P. Lv et al., "Microalterations of esophagus in patients with non-erosive reflux disease: in-vivo diagnosis by confocal laser endomicroscopy and its relationship with gastroesophageal reflux," The American Journal of Gastroenterology, vol. 107, no. 6, pp. 864-874, 2012.

[37] Y. Kakeji, S. Yamaguchi, D. Yoshida et al., "Development and assessment of morphologic criteria for diagnosing gastric cancer using confocal endomicroscopy: an ex vivo and in vivo study," Endoscopy, vol. 38, no. 9, pp. 886-890, 2006.

[38] S. Kitabatake, Y. Niwa, R. Miyahara et al., "Confocal endomicroscopy for the diagnosis of gastric cancer in vivo," Endoscopy, vol. 38, no. 11, pp. 1110-1114, 2006.

[39] H. Liu, Y. Q. Li, T. Yu et al., "Confocal endomicroscopy for in vivo detection of microvascular architecture in normal and malignant lesions of upper gastrointestinal tract," Journal of Gastroenterology and Hepatology, vol. 23, no. 1, pp. 56-61, 2008.

[40] C. Gheorghe, R. Iacob, M. Dumbrava, G. Becheanu, and M. Ionescu, "Confocal laser endomicroscopy and ultrasound endoscopy during the same endoscopic session for diagnosis and staging of gastric neoplastic lesions," Chirurgia, vol. 104, no. 1, pp. 17-24, 2009.

[41] K. Banno, Y. Niwa, R. Miyahara et al., "Confocal endomicroscopy for phenotypic diagnosis of gastric cancer," Journal of Gastroenterology and Hepatology, vol. 25, no. 4, pp. 712-718, 2010.

[42] W. B. Li, X. L. Zuo, F. Zuo et al., "Characterization and identification of gastric hyperplastic polyps and adenomas by confocal laser endomicroscopy," Surgical Endoscopy, vol. 24, no. 3, pp. 517-524, 2010.

[43] Z. Li, T. Yu, X. L. Zuo et al., "Confocal laser endomicroscopy for in vivo diagnosis of gastric intraepithelial neoplasia: a feasibility study," Gastrointestinal Endoscopy, vol. 72, no. 6, pp. 1146-1153, 2010.

[44] S. R. Jeon, W. Y. Cho, S. Y. Jin, Y. K. Cheon, S. R. Choi, and J. Y. Cho, "Optical biopsies by confocal endomicroscopy prevent additive endoscopic biopsies before endoscopic submucosal dissection in gastric epithelial neoplasias: a prospective, comparative study," Gastrointestinal Endoscopy, vol. 74, no. 4, pp. 772-780, 2011.

[45] R. Ji, X.-L. Zuo, C.-Q. Li, C.-J. Zhou, and Y.-Q. Li, “Confocal endomicroscopy for in vivo prediction of completeness after endoscopic mucosal resection," Surgical Endoscopy, vol. 25, no. 6, pp. 1933-1938, 2011.

[46] W.-B. Li, X.-L. Zuo, C.-Q. Li et al., "Diagnostic value of confocal laser endomicroscopy for gastric superficial cancerous lesions," Gut, vol. 60, no. 3, pp. 299-306, 2011.

[47] L. G. Lim, K. G. Yeoh, M. Salto-Tellez et al., "Experienced versus inexperienced confocal endoscopists in the diagnosis of gastric adenocarcinoma and intestinal metaplasia on confocal images," Gastrointestinal Endoscopy, vol. 73, no. 6, pp. 1141-1147, 2011.

[48] S. F. Wang, Y. S. Yang, L. X. Wei et al., "Diagnosis of gastric intraepithelial neoplasia by narrow-band imaging and confocal laser endomicroscopy," World Journal of Gastroenterology, vol. 18, no. 34, pp. 4771-4780, 2012.
[49] G. H. Bok, S. R. Jeon, J. Y. Cho et al., "The accuracy of probebased confocal endomicroscopy versus conventional endoscopic biopsies for the diagnosis of superficial gastric neoplasia (with videos)," Gastrointestinal Endoscopy, vol. 77, no. 6, pp. 899-908, 2013.

[50] Y. T. Guo, Y. Q. Li, T. Yu et al., "Diagnosis of gastric intestinal metaplasia with confocal laser endomicroscopy in vivo: a prospective study," Endoscopy, vol. 40, no. 7, pp. 547-553, 2008.

[51] R. Ji, T. Yu, X. M. Gu et al., "Gastric metaplasia of the duodenum: in vivo diagnosis by endomicroscopy and its relationship with functional dyspepsia," Journal of Gastroenterology and Hepatology, vol. 26, no. 1, pp. 73-77, 2011.

[52] R. Ji, X. L. Zuo, T. Yu et al., "Mucosal barrier defects in gastric intestinal metaplasia: in vivo evaluation by confocal endomicroscopy," Gastrointestinal Endoscopy, vol. 75, no. 5, pp. 980-987, 2012.

[53] L. G. Lim, K. G. Yeoh, S. Srivastava, Y. H. Chan, M. Teh, and K. Y. Ho, "Comparison of probe-based confocal endomicroscopy with virtual chromoendoscopy and white-light endoscopy for diagnosis of gastric intestinal metaplasia," Surgical Endoscopy, vol. 27, no. 12, pp. 4649-4655, 2013.

[54] R. Pittayanon, R. Rerknimitr, N. Wisedopas et al., "Flexible spectral imaging color enhancement plus probe-based confocal laser endomicroscopy for gastric intestinal metaplasia detection," Journal of Gastroenterology and Hepatology, vol. 28, no. 6, pp. 1004-1009, 2013.

[55] Z. Li, X. L. Zuo, T. Yu et al., "Confocal laser endomicroscopy for in vivo detection of gastric intestinal metaplasia: a randomized controlled trial," Endoscopy, vol. 46, no. 4, pp. 282-290, 2014.

[56] T. Liu, H. Zheng, W. Gong, C. Chen, and B. Jiang, “The accuracy of confocal laser endomicroscopy, narrow band imaging, and chromoendoscopy for the detection of atrophic gastritis," Journal of Clinical Gastroenterology, vol. 49, no. 5, pp. 379-386, 2015.

[57] R. Ji, Y. Q. Li, X. M. Gu, T. Yu, X. L. Zuo, and C. J. Zhou, "Confocal laser endomicroscopy for diagnosis of Helicobacter pylori infection: a prospective study," Journal of Gastroenterology and Hepatology, vol. 25, no. 4, pp. 700-705, 2010.

[58] P. Wang, R. Ji, T. Yu et al., "Classification of histological severity of Helicobacter pylori-associated gastritis by confocal laser endomicroscopy," World Journal of Gastroenterology, vol. 16, no. 41, pp. 5203-5210, 2010.

[59] L. G. Lim, J. Neumann, T. Hansen et al., "Confocal endomicroscopy identifies loss of local barrier function in the duodenum of patients with Crohn's disease and ulcerative colitis," Inflammatory Bowel Diseases, vol. 20, no. 5, pp. 892-900, 2014.

[60] R. W. L. Leong, N. Q. Nguyen, C. G. Meredith et al., "In vivo confocal endomicroscopy in the diagnosis and evaluation of celiac disease," Gastroenterology, vol. 135, no. 6, pp. 1870-1876, 2008.

[61] U. Günther, S. Daum, F. Heller et al., "Diagnostic value of confocal endomicroscopy in celiac disease," Endoscopy, vol. 42, no. 3, pp. 197-202, 2010.

[62] K. Venkatesh, A. Abou-Taleb, M. Cohen et al., "Role of confocal endomicroscopy in the diagnosis of celiac disease," Journal of Pediatric Gastroenterology \& Nutrition, vol. 51, no. 3, pp. 274279, 2010.

[63] O. Watanabe, T. Ando, O. Maeda et al., "Confocal endomicroscopy in patients with ulcerative colitis," Journal of Gastroenterology and Hepatology, vol. 23, supplement 2, pp. S286-S290, 2008. 
[64] C. Trovato, A. Sonzogni, G. Fiori et al., "Confocal laser endomicroscopy for the detection of mucosal changes in ileal pouch after restorative proctocolectomy," Digestive and Liver Disease, vol. 41, no. 8, pp. 578-585, 2009.

[65] C. Q. Li, X. J. Xie, T. Yu et al., "Classification of inflammation activity in ulcerative colitis by confocal laser endomicroscopy," The American Journal of Gastroenterology, vol. 105, no. 6, pp. 1391-1396, 2010.

[66] J. J. Liu, K. Wong, A. L. Thiesen et al., "Increased epithelial gaps in the small intestines of patients with inflammatory bowel disease: density matters," Gastrointestinal Endoscopy, vol. 73, no. 6, pp. 1174-1180, 2011.

[67] D. Moussata, M. Goetz, A. Gloeckner et al., "Confocal laser endomicroscopy is a new imaging modality for recognition of intramucosal bacteria in inflammatory bowel disease in vivo," Gut, vol. 60, no. 1, pp. 26-33, 2011.

[68] R. Kiesslich, C. A. Duckworth, D. Moussata et al., "Local barrier dysfunction identified by confocal laser endomicroscopy predicts relapse in inflammatory bowel disease," Gut, vol. 61, no. 8, pp. 1146-1153, 2012.

[69] E. Krauss, A. Agaimy, H. Neumann et al., "Characterization of lymphoid follicles with red ring signs as first manifestation of early Crohn's disease by conventional histopathology and confocal laser endomicroscopy," International Journal of Clinical and Experimental Pathology, vol. 5, no. 5, pp. 411-421, 2012.

[70] H. Neumann, M. Vieth, R. Atreya et al., "Assessment of Crohn's disease activity by confocal laser endomicroscopy," Inflammatory Bowel Diseases, vol. 18, no. 12, pp. 2261-2269, 2012.

[71] J.-F. Turcotte, K. Wong, S. J. Mah et al., "Increased epithelial gaps in the small intestine are predictive of hospitalization and surgery in patients with inflammatory bowel disease," Clinical and Translational Gastroenterology, vol. 3, article e19, 2012.

[72] N. Musquer, S. Coquenlorge, A. Bourreille et al., "Probe-based confocal laser endomicroscopy: a new method for quantitative analysis of pit structure in healthy and Crohn's disease patients," Digestive and Liver Disease, vol. 45, no. 6, pp. 487-492, 2013.

[73] R. Atreya, H. Neumann, C. Neufert et al., "In vivo imaging using fluorescent antibodies to tumor necrosis factor predicts therapeutic response in Crohn's disease," Nature Medicine, vol. 20, no. 3, pp. 313-318, 2014.

[74] A. Buda, G. Hatem, H. Neumann et al., "Confocal laser endomicroscopy for prediction of disease relapse in ulcerative colitis: a pilot study," Journal of Crohn's and Colitis, vol. 8, no. 4, pp. 304-311, 2014.

[75] C.-Q. Li, J. Liu, R. Ji, Z. Li, X.-J. Xie, and Y.-Q. Li, "Use of confocal laser endomicroscopy to predict relapse of ulcerative colitis," BMC Gastroenterology, vol. 14, article 45, 2014.

[76] D. P. Hurlstone, M. Thomson, S. Brown, N. Tiffin, S. S. Cross, and M. D. Hunter, "Confocal endomicroscopy in ulcerative colitis: differentiating dysplasia-associated lesional mass and adenoma-like mass," Clinical Gastroenterology and Hepatology, vol. 5, no. 10, pp. 1235-1241, 2007.

[77] R. Kiesslich, M. Goetz, K. Lammersdorf et al., "Chromoscopyguided endomicroscopy increases the diagnostic yield of intraepithelial neoplasia in ulcerative colitis," Gastroenterology, vol. 132, no. 3, pp. 874-882, 2007.

[78] U. Günther, D. Kusch, F. Heller et al., "Surveillance colonoscopy in patients with inflammatory bowel disease: comparison of random biopsy vs. targeted biopsy protocols," International Journal of Colorectal Disease, vol. 26, no. 5, pp. 667-672, 2011.

[79] T. Hlavaty, M. Huorka, T. Koller et al., "Colorectal cancer screening in patients with ulcerative and Crohn's colitis with use of colonoscopy, chromoendoscopy and confocal endomicroscopy," European Journal of Gastroenterology and Hepatology, vol. 23, no. 8, pp. 680-689, 2011.

[80] F. J. C. van den Broek, J. A. van Es, S. van Eeden et al., "Pilot study of probe-based confocal laser endomicroscopy during colonoscopic surveillance of patients with longstanding ulcerative colitis," Endoscopy, vol. 43, no. 2, pp. 116-122, 2011.

[81] A. Rispo, F. Castiglione, S. Staibano et al., "Diagnostic accuracy of confocal laser endomicroscopy in diagnosing dysplasia in patients affected by long-standing ulcerative colitis," World Journal of Gastrointestinal Endoscopy, vol. 4, no. 9, pp. 414-420, 2012.

[82] R. Kiesslich, J. Burg, M. Vieth et al., "Confocal laser endoscopy for diagnosing intraepithelial neoplasias and colorectal cancer in vivo," Gastroenterology, vol. 127, no. 3, pp. 706-713, 2004.

[83] I. Odagi, T. Kato, H. Imazu, M. Kaise, S. Omar, and H. Tajiri, "Examination of normal intestine using confocal endomicroscopy," Journal of Gastroenterology and Hepatology, vol. 22, no. 5, pp. 658-662, 2007.

[84] T. D. Wang, S. Friedland, P. Sahbaie et al., "Functional imaging of colonic mucosa with a fibered confocal microscope for real-time in vivo pathology," Clinical Gastroenterology and Hepatology, vol. 5, no. 11, pp. 1300-1305, 2007.

[85] A. M. Buchner, M. W. Shahid, M. G. Heckman et al., "Comparison of probe-based confocal laser endomicroscopy with virtual chromoendoscopy for classification of colon polyps," Gastroenterology, vol. 138, no. 3, pp. 834-842, 2010.

[86] G. D. De Palma, S. Staibano, S. Siciliano et al., "In vivo characterisation of superficial colorectal neoplastic lesions with high-resolution probe-based confocal laser endomicroscopy in combination with video-mosaicing: a feasibility study to enhance routine endoscopy," Digestive and Liver Disease, vol. 42, no. 11, pp. 791-797, 2010.

[87] V. Gómez, A. M. Buchner, E. Dekker et al., "Interobserver agreement and accuracy among international experts with probebased confocal laser endomicroscopy in predicting colorectal neoplasia," Endoscopy, vol. 42, no. 4, pp. 286-291, 2010.

[88] S. Sanduleanu, A. Driessen, E. Gomez-Garcia, W. Hameeteman, A. de Bruïne, and A. Masclee, "In vivo diagnosis and classification of colorectal neoplasia by chromoendoscopy-guided confocal laser endomicroscopy," Clinical Gastroenterology and Hepatology, vol. 8, no. 4, pp. 371-378, 2010.

[89] X. J. Xie, C. Q. Li, X. L. Zuo et al., "Differentiation of colonic polyps by confocal laser endomicroscopy," Endoscopy, vol. 43, no. 2, pp. 87-93, 2011.

[90] B. André, T. Vercauteren, A. M. Buchner, M. Krishna, N. Ayache, and M. B. Wallac, "Software for automated classification of probe-based confocal laser endomicroscopy videos of colorectal polyps," World Journal of Gastroenterology, vol. 18, no. 39, pp. 5560-5569, 2012.

[91] T. Cârţână, A. Săftoiu, L. G. Gruionu et al., "Confocal laser endomicroscopy for the morphometric evaluation of microvessels in human colorectal cancer using targeted anti-CD31 antibodies," PLoS ONE, vol. 7, no. 12, Article ID e52815, 2012.

[92] E. Coron, J. F. Mosnier, A. Ahluwalia et al., "Colonic mucosal biopsies obtained during confocal endomicroscopy are prestained with fluorescein in vivo and are suitable for histologic evaluation," Endoscopy, vol. 44, no. 2, pp. 148-153, 2012.

[93] T. Kuiper, F. J. C. van den Broek, S. van Eeden, P. Fockens, and E. Dekker, "Feasibility and accuracy of confocal endomicroscopy in comparison with narrow-band imaging and chromoendoscopy for the differentiation of colorectal lesions," The 
American Journal of Gastroenterology, vol. 107, no. 4, pp. 543$550,2012$.

[94] M. Mascolo, S. Staibano, G. Ilardi et al., "Probe-based confocal laser endomicroscopy evaluation of colon preneoplastic lesions, with particular attention to the aberrant crypt foci, and comparative assessment with histological features obtained by conventional endoscopy," Gastroenterology Research and Practice, vol. 2012, Article ID 645173, 6 pages, 2012.

[95] M. W. Shahid, A. M. Buchner, M. Raimondo, T. A. Woodward, M. Krishna, and M. B. Wallace, "Accuracy of real-time vs. blinded offline diagnosis of neoplastic colorectal polyps using probe-based confocal laser endomicroscopy: a pilot study," Endoscopy, vol. 44, no. 4, pp. 343-348, 2012.

[96] M. W. Shahid, A. M. Buchner, M. G. Heckman et al., "Diagnostic accuracy of probe-based confocal laser endomicroscopy and narrow band imaging for small colorectal polyps: a feasibility study," The American Journal of Gastroenterology, vol. 107, no. 2, pp. 231-239, 2012.

[97] M. W. Shahid, A. M. Buchner, E. Coron et al., "Diagnostic accuracy of probe-based confocal laser endomicroscopy in detecting residual colorectal neoplasia after EMR: a prospective study," Gastrointestinal Endoscopy, vol. 75, no. 3, pp. 525-533, 2012.

[98] V. Gómez, M. W. Shahid, M. Krishna, M. G. Heckman, J. E. Crook, and M. B. Wallace, "Classification criteria for advanced adenomas of the colon by using probe-based confocal laser endomicroscopy: a preliminary study," Diseases of the Colon and Rectum, vol. 56, no. 8, pp. 967-973, 2013.

[99] C. Liu, C. Q. Li, X. L. Zuo et al., "Confocal laser endomicroscopy for the diagnosis of colorectal cancer in vivo," Journal of Digestive Diseases, vol. 14, no. 5, pp. 259-265, 2013.

[100] J. Liu, X. Zuo, C. Li et al., "In vivo molecular imaging of epidermal growth factor receptor in patients with colorectal neoplasia using confocal laser endomicroscopy," Cancer Letters, vol. 330, no. 2, pp. 200-207, 2013.

[101] A. Ciocâlteu, A. Săftoiu, T. Cârţână et al., "Evaluation of new morphometric parameters of neoangiogenesis in human colorectal cancer using confocal laser endomicroscopy (CLE) and targeted panendothelial markers," PLoS ONE, vol. 9, no. 3, Article ID e91084, 2014.

[102] X. M. Yuan, Z. Li, R. Ji et al., "Minimal influence of expertise on the evaluation of colorectal neoplastic lesions by confocal laser endomicroscopy," Journal of Gastroenterology and Hepatology, vol. 29, no. 1, pp. 91-95, 2014.

[103] C. Bojarski, U. Günther, K. Rieger et al., "In vivo diagnosis of acute intestinal graft-versus-host disease by confocal endomicroscopy," Endoscopy, vol. 41, no. 5, pp. 433-438, 2009.

[104] H. Neumann, C. Günther, M. Vieth et al., "Confocal laser endomicroscopy for in vivo diagnosis of Clostridium difficile associated colitis-a pilot study," PLoS ONE, vol. 8, no. 3, Article ID e58753, 2013.

[105] J.-F. Turcotte, D. Kao, S. J. Mah et al., "Breaks in the wall: increased gaps in the intestinal epithelium of irritable bowel syndrome patients identified by confocal laser endomicroscopy (with videos)," Gastrointestinal Endoscopy, vol. 77, no. 4, pp. 624-630, 2013.

[106] A. Fritscher-Ravens, D. Schuppan, M. Ellrichmann et al., "Confocal endomicroscopy shows food-associated changes in the intestinal mucosa of patients with irritable bowel syndrome," Gastroenterology, vol. 147, no. 5, pp. 1012-1020.e4, 2014.

[107] A. Meining, E. Frimberger, V. Becker et al., "Detection of cholangiocarcinoma in vivo using miniprobe-based confocal fluorescence microscopy," Clinical Gastroenterology and Hepatology, vol. 6, no. 9, pp. 1057-1060, 2008.

[108] M. Giovannini, E. Bories, G. Monges, C. Pesenti, F. Caillol, and J. R. Delpero, "Results of a phase I-II study on intraductal confocal microscopy (IDCM) in patients with common bile duct (CBD) stenosis," Surgical Endoscopy, vol. 25, no. 7, pp. 2247-2253, 2011.

[109] A. Meining, Y. K. Chen, D. Pleskow et al., "Direct visualization of indeterminate pancreaticobiliary strictures with probe-based confocal laser endomicroscopy: a multicenter experience," Gastrointestinal Endoscopy, vol. 74, no. 5, pp. 961-968, 2011.

[110] A. Meining, R. J. Shah, A. Slivka et al., "Classification of probebased confocal laser endomicroscopy findings in pancreaticobiliary strictures," Endoscopy, vol. 44, no. 3, pp. 251-257, 2012.

[111] F. Caillol, B. Filoche, M. Gaidhane, and M. Kahaleh, "Refined probe-based confocal laser endomicroscopy classification for biliary strictures: the Paris classification," Digestive Diseases and Sciences, vol. 58, no. 6, pp. 1784-1789, 2013.

[112] F. Caillol, E. Bories, F. Poizat et al., "Endomicroscopy in bile duct: inflammation interferes with pCLE applied in the bile duct: a prospective study of 54 patients," United European Gastroenterology Journal, vol. 1, no. 2, pp. 120-127, 2013.

[113] M. Heif, R. D. Yen, and R. J. Shah, "ERCP with probe-based confocal laser endomicroscopy for the evaluation of dominant biliary stenoses in primary sclerosing cholangitis patients," Digestive Diseases and Sciences, vol. 58, no. 7, pp. 2068-2074, 2013.

[114] F. Caillol, E. Bories, A. Autret et al., "Evaluation of pCLE in the bile duct: final results of EMID study: pCLE: impact in the management of bile duct strictures," Surgical Endoscopy, vol. 29, no. 9, pp. 2661-2668, 2015.

[115] A. Slivka, I. Gan, P. Jamidar et al., "Validation of the diagnostic accuracy of probe-based confocal laser endomicroscopy for the characterization of indeterminate biliary strictures: results of a prospective multicenter international study," Gastrointestinal Endoscopy, vol. 81, no. 2, pp. 282-290, 2015.

[116] M. Kahaleh, B. G. Turner, K. Bezak et al., "Probe-based confocal laser endomicroscopy in the pancreatic duct provides direct visualization of ductal structures and aids in clinical management," Digestive and Liver Disease, vol. 47, no. 3, pp. 202204, 2015.

[117] Y. Nakai, T. Iwashita, D. H. Park, J. B. Samarasena, J. G. Lee, and K. J. Chang, "Diagnosis of pancreatic cysts: EUSguided, through-the-needle confocal laser-induced endomicroscopy and cystoscopy trial: DETECT study," Gastrointestinal Endoscopy, vol. 81, no. 5, pp. 1204-1214, 2015.

[118] B. Napoléon, A.-I. Lemaistre, B. Pujol et al., "A novel approach to the diagnosis of pancreatic serous cystadenoma: needle-based confocal laser endomicroscopy," Endoscopy, vol. 47, no. 1, pp. 26-32, 2015.

[119] D. P. Hurlstone and D. S. Sanders, "Recent advances in chromoscopic colonoscopy and endomicroscopy," Current Gastroenterology Reports, vol. 8, no. 5, pp. 409-415, 2006.

[120] A. Meining, D. Saur, M. Bajbouj et al., "In vivo histopathology for detection of gastrointestinal neoplasia with a portable, confocal miniprobe: an examiner blinded analysis," Clinical Gastroenterology and Hepatology, vol. 5, no. 11, pp. 1261-1267, 2007.

[121] P. Sharma, T. J. Savides, M. I. Canto et al., "The American Society for Gastrointestinal Endoscopy PIVI (Preservation and Incorporation of Valuable Endoscopic Innovations) on imaging in Barrett's Esophagus," Gastrointestinal Endoscopy, vol. 76, no. 2, pp. 252-254, 2012. 
[122] A. Gupta, B. M. Attar, P. Koduru, A. R. Murali, B. T. Go, and R. Agarwal, "Utility of confocal laser endomicroscopy in identifying high-grade dysplasia and adenocarcinoma in Barrett's esophagus: a systematic review and meta-analysis," European Journal of Gastroenterology and Hepatology, vol. 26, no. 4, pp. 369-377, 2014.

[123] J. N. Zhang, Y. Q. Li, Y. A. Zhao et al., "Classification of gastric pit patterns by confocal endomicroscopy," Gastrointestinal Endoscopy, vol. 67, no. 6, pp. 843-853, 2008.

[124] C. S. Loeser, M. E. Robert, A. Mennone, M. H. Nathanson, and P. Jamidar, "Confocal endomicroscopic examination of malignant biliary strictures and histologic correlation with lymphatics," Journal of Clinical Gastroenterology, vol. 45, no. 3, pp. 246-252, 2011.

[125] J. P. Talreja, B. G. Turner, F. G. Gress et al., "Pre- and posttraining session evaluation for interobserver agreement and diagnostic accuracy of probe-based confocal laser endomicroscopy for biliary strictures," Digestive Endoscopy, vol. 26, no. 4, pp. 577-580, 2014

[126] M. B. Wallace and P. Fockens, "Probe-based confocal laser endomicroscopy," Gastroenterology, vol. 136, no. 5, pp. 15091513, 2009.

[127] M. De Bellis, S. Sherman, E. L. Fogel et al., "Tissue sampling at ERCP in suspected malignant biliary strictures (Part 1)," Gastrointestinal Endoscopy, vol. 56, no. 4, pp. 552-561, 2002.

[128] M. A. Anderson, V. Appalaneni, T. Ben-Menachem et al., “The role of endoscopy in the evaluation and treatment of patients with biliary neoplasia," Gastrointestinal Endoscopy, vol. 77, no. 2, pp. 167-174, 2013.

[129] W. R. Brugge, K. Lewandrowski, E. Lee-Lewandrowski et al., "Diagnosis of pancreatic cystic neoplasms: a report of the cooperative pancreatic cyst study," Gastroenterology, vol. 126, no. 5, pp. 1330-1336, 2004. 


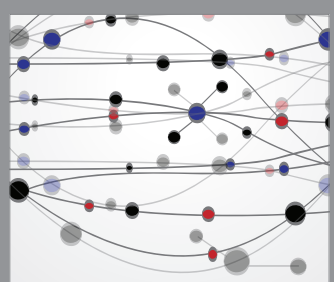

The Scientific World Journal
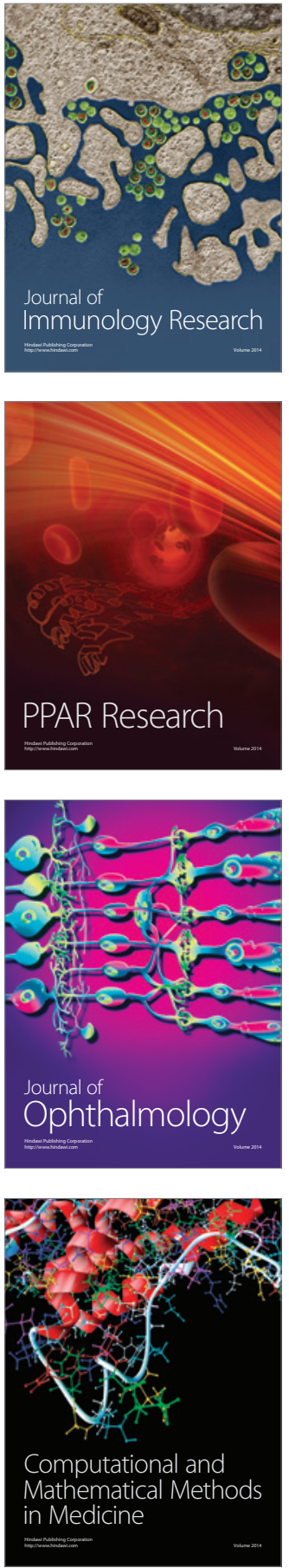

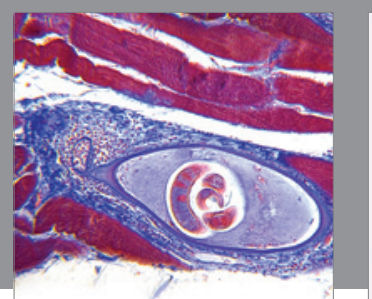

Gastroenterology Research and Practice

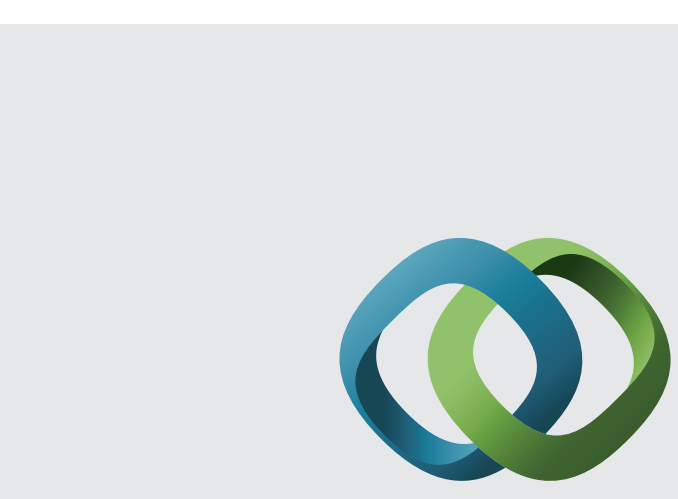

\section{Hindawi}

Submit your manuscripts at

http://www.hindawi.com
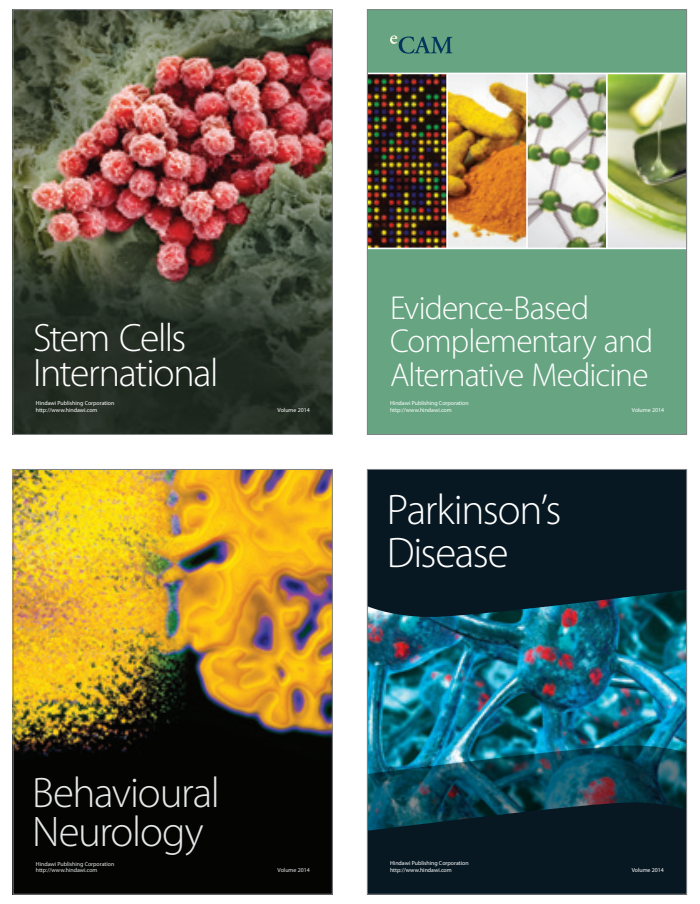
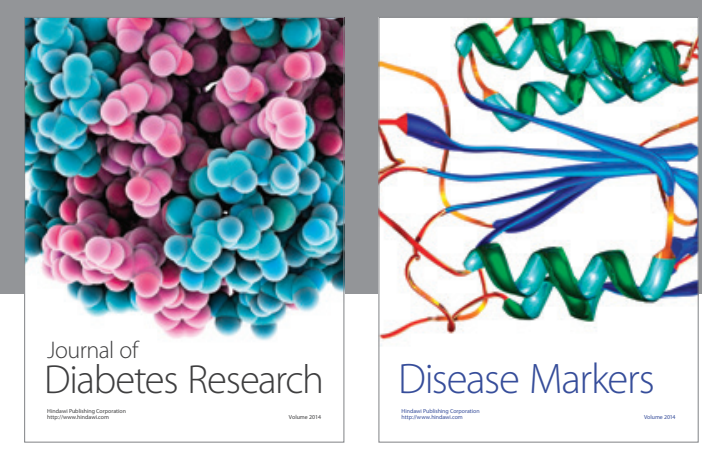

Disease Markers
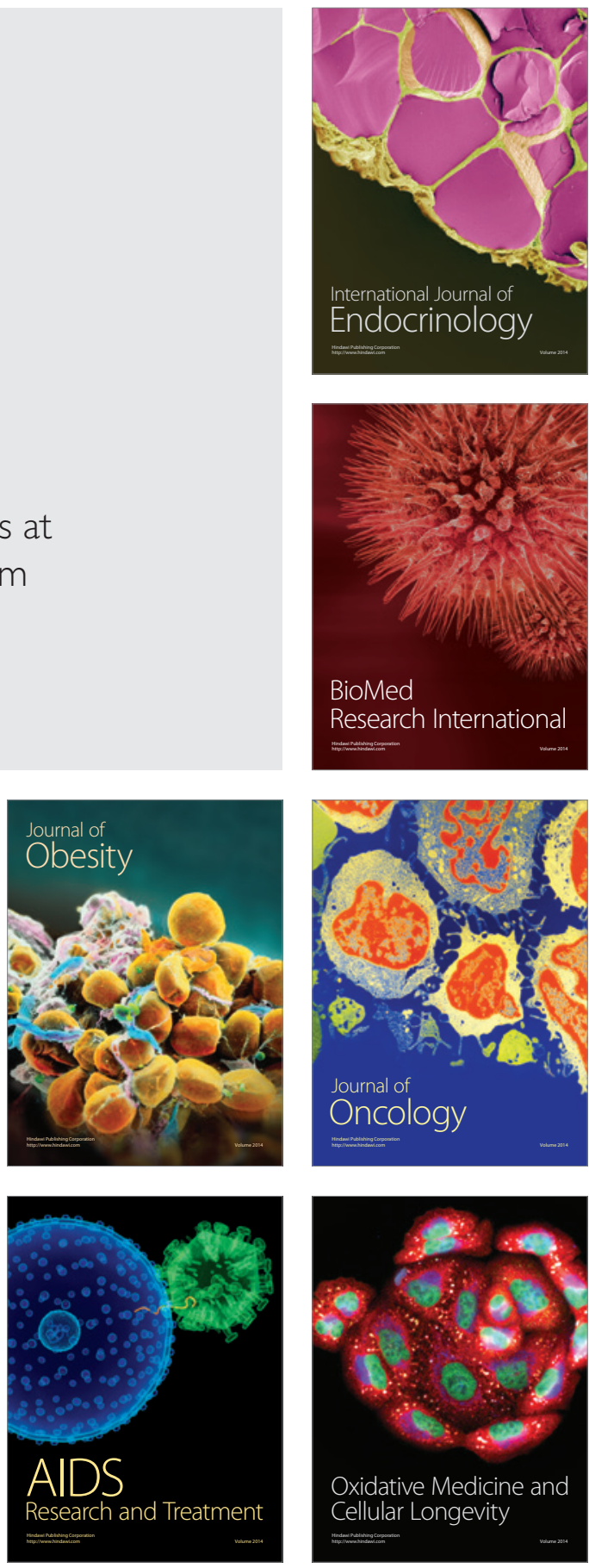